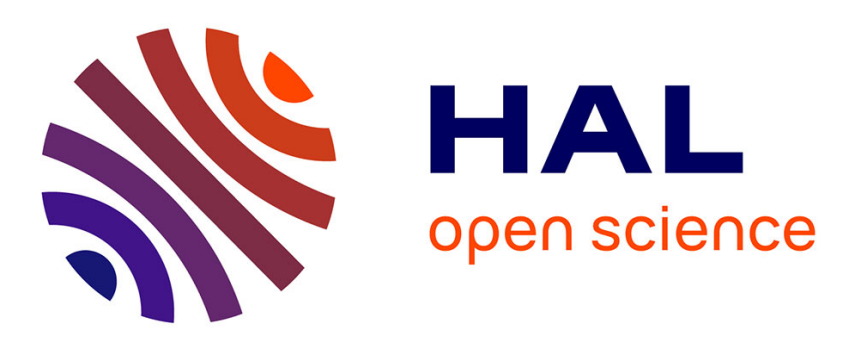

\title{
Backward sensitivity analysis and reduced-order covariance estimation in noninvasive parameter identification for cerebral arteries
}

\author{
Robert Rapadamnaba, Franck Nicoud, Bijan Mohammadi
}

\section{To cite this version:}

Robert Rapadamnaba, Franck Nicoud, Bijan Mohammadi. Backward sensitivity analysis and reduced-order covariance estimation in noninvasive parameter identification for cerebral arteries. International Journal for Numerical Methods in Biomedical Engineering, 2019, 35 (4), pp.e3170. 10.1002/cnm.3170 . hal-01958244

\author{
HAL Id: hal-01958244 \\ https://hal.science/hal-01958244
}

Submitted on 17 Dec 2018

HAL is a multi-disciplinary open access archive for the deposit and dissemination of scientific research documents, whether they are published or not. The documents may come from teaching and research institutions in France or abroad, or from public or private research centers.
L'archive ouverte pluridisciplinaire HAL, est destinée au dépôt et à la diffusion de documents scientifiques de niveau recherche, publiés ou non, émanant des établissements d'enseignement et de recherche français ou étrangers, des laboratoires publics ou privés. 


\title{
Backward sensitivity analysis and reduced-order covariance estimation in noninvasive parameter identification for cerebral arteries
}

\author{
Robert Rapadamnaba $^{1, *}$, Franck Nicoud $^{1}$ and Bijan Mohammadi ${ }^{1}$ \\ ${ }^{1}$ IMAG, Université de Montpellier, CC051, 34095 Montpellier, France.
}

\begin{abstract}
SUMMARY
Using a previously developed inversion platform for functional cerebral medical imaging with ensemble Kalman filters, this work analyzes the sensitivity of the results with respect to different parameters entering the physical model and inversion procedure, such as the inlet flow rate from the heart, the choice of the boundary conditions, and the nonsymmetry in the network terminations. It also proposes an alternative low complexity construction for the covariance matrix of the hemodynamic parameters of a network of arteries including the circle of Willis. The platform takes as input patient-specific blood flow rates extracted from magnetic resonance angiography and magnetic resonance imaging (dicom files) and is applied to several available patients data. The paper presents full analysis of the results for one of these patients, including a sensitivity study with respect to the proximal and distal boundary conditions. The results notably show that the uncertainties on the inlet flow rate led to uncertainties of the same order of magnitude in the estimated parameters (blood pressure and elastic parameters) and that three-lumped parameters boundary conditions are necessary for a correct retrieval of the target signals.
\end{abstract}

KEY WORDS: covariance matrix, ensemble Kalman filter, parameter estimation, reduced order compartment blood model, uncertainty quantification, sensitivity analysis. 


\section{INTRODUCTION}

Cardiovascular diseases are obviously major health concerns nowadays as according to the World Health Organization, they are the leading cause of death globally [1]. Among these diseases, intracranial aneurysms, which usually take place in the circle of Willis (CoW) [2], require special attention. Indeed, according to the Brain Aneurysm Foundation, the cerebral aneurysm is known as silent killer because in the most of the cases, it is completely asymptomatic and is discovered by chance frequently in people undergoing brain imaging, such as magnetic resonance angiography and magnetic resonance imaging (MRA\&MRI), and for other reasons, such as evaluation of headaches, after head trauma, or in work-up of other neurological symptoms [3]. Often in these situations, the aneurysm itself is an incidental finding, unrelated to the symptoms that prompted the imaging, but a ruptured aneurysm can cause life-threatening blood loss, which leads to death. That is why over the last few decades, to better understand and identify the mechanisms linked to aneurysm formation and rupture, many researchers, across a series of studies, made every effort to establish the key factors that contribute to the development and the rupture of cerebral aneurysms [4-9]. Some addressed the substantial challenges facing them regarding hemodynamics and the blood flow mechanism in the CoW [10-13]. Their main objective was to understand the factors increasing the risk of stroke and the blood flow distribution in the brain. Others have demonstrated the considerable influence of patient-specific anatomy of cerebral arterial network on blood flow patterns in local cerebral aneurysms [10, 14-19].

In previous studies [20-24], still others have developed an algorithm based on a data assimilation technique to estimate hemodynamic parameters such as elastic properties of arteries, arterial compliance, and boundary condition parameters (Windkessel boundary parameters and reflection coefficients).

\footnotetext{
${ }^{*}$ Correspondence to: Robert Rapadamnaba, IMAG, Université de Montpellier, CC051, 34095 Montpellier, France. Email: robert.rapadamnaba@umontpellier.fr
} 
In our recent publications $[25,26]$, the usefulness of this kind of algorithm in estimating the hemodynamic parameters (eg, the wall thickness and the Young modulus), which are difficult to identify noninvasively, was demonstrated. And to examine the robustness of the approach, several sensitivity analyses have been carried out. More precisely, the behavior of the algorithm has been analyzed for

- different initial guesses for the parameters,

- different levels of observations uncertainty,

- the effect of bias in known parameter values and the type of observations,

- the effect of the ensemble size $q_{\text {ens }}$ on the estimated parameters,

- the location of observations and the number of observations $n_{\text {obs }}$.

This work considers other sources of uncertainties and studies their impacts on the inversion outcomes. More precisely, it will address

- the uncertainties on the inlet flow rate time series from MRA\&MRI, which has been considered as deterministic in our previous works;

- the uncertainties related to the choice of the boundary conditions at the terminations;

- the uncertainties related to the symmetry assumption previously used between the left and right network terminations.

Another proposal in this paper is a deterministic construction for backward uncertainty propagation not using an ensemble approach and an alternative estimation of the covariance matrix of the estimated parameters after inversion. This construction can be used even without an ensemble formulation and with deterministic gradient-based minimization algorithms.

The general minimization framework in which the fluid-structure coupling is cast is recalled in Section 2. The cardiovascular network model, which is made of OD lumped compartments, is presented together with the associated minimization problem in Section 3. Section 4 presents the set up, which will be used to illustrate the different results. This is from one of the patient-specific data provided by Montpellier University Hospital, Gui de Chauliac. Section 5 gathers the results 
of various sensitivity analyses. The inversion procedure is based on the ensemble Kalman filter (EnKF) algorithm described in Lal et al. [25]. These ensemble algorithms give an estimation of the covariance matrix $\operatorname{cov}_{\mathbf{x}}$ of the optimization parameters. This is compared to an alternative derivation of the covariance matrix in Section 6. Section 7 discusses some limitations of the paper in the light of the model assumptions.

\section{GENERAL SETTINGS}

This section presents materials for this work. It follows in broad outline the description made in Mohammadi [27].

This study focuses on a class of minimization problems written as follows:

$$
\min _{\mathbf{x} \in \mathbf{O}_{a d}} J\left(\mathbf{y}(\mathbf{x}, \mathbf{z}), \mathbf{y}_{\text {obs }}\right), \quad \mathbf{y}, \mathbf{y}_{\text {obs }} \in \mathcal{Y} \subset \mathbb{R}^{n_{o b s}}, \mathbf{z} \in \mathcal{Z} \subset \mathbb{R}^{m}, \mathbf{x} \in \mathbf{O}_{a d} \subset \mathbb{R}^{n}
$$

where $\mathbf{x}, \mathbf{y}_{\mathbf{o b s}}$, and $\mathbf{z}$ are independent variables. Only the state variable, $\mathbf{y}$, depends on the cost function, $J$. The optimization parameter $\mathbf{x}$ belongs to $\mathbf{O}_{a d}$ the optimization admissible domain [28]. The physical meaning of all the variables will be given in Section 3.2.

This is a very general context, and it is important to address the effects of the variability in $\mathbf{z}$ and $\mathbf{y}_{\text {obs }}$ on the solution of the minimization problem. To analyze these, different approaches will be adopted: an ensemble approach (ie, EnKF here) for the effect of the variability in $\mathbf{y}_{\mathbf{o b s}}$ and a separated treatment through a consideration of adequate ensemble of ensembles for the effect of the variability in $\mathbf{z}$.

To be more precise, attention is paid on functional $J$ of the form

$$
J\left(\mathbf{y}(\mathbf{x}, \mathbf{z}), \mathbf{y}_{\text {obs }}\right)=\left\|\mathbf{y}(\mathbf{x}, \mathbf{z})-\mathbf{y}_{\text {obs }}\right\|_{*},
$$

where the state $\mathbf{y}(\mathbf{x}, \mathbf{z})$ is solution of a state equation $F(\mathbf{y}(\mathbf{x}, \mathbf{z}))=0, \mathbf{y}_{\text {obs }}$ comes from a direct observation (measurements) of the system, and $\|\cdot\|_{*}$ is a suitable norm. These will be specified in Section 3.2. The variable $\mathbf{y}_{\mathbf{o b s}}$ is assumed uncertain and independent, and its components are given by their probability density functions here assumed Gaussian $\mathcal{N}\left(\mu_{i}, \sigma_{i}^{2}\right), i=1, \ldots, n_{\text {obs }}$ with means $\mu_{i}$ and variances $\sigma_{i}^{2}$. 


\section{MODELLING AND PROBLEM SPECIFICATION}

\subsection{Physical model}

Many sophisticated and complex physical models representing the human cardiovascular system exist in the literature [10,29-32], the simplest of these being the OD model (also called compartement model or lumped parameter model). It is the physical model adopted for this work. In this model, built using an electrical analogy [32-35], the arterial network is divided into different compartments, each with a resistor (resistance $R$ of blood due to blood viscosity and the vessel diameter), an inductor (blood inertia $L$ ), and a capacitor (compliance $C$ of the artery corresponding to the quality of the latter to accumulate and release blood due to elastic deformations) as shown in Figure 1.

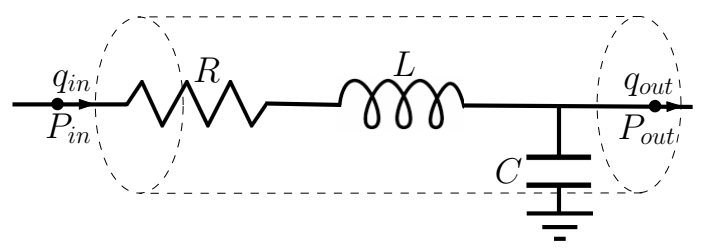

Figure 1. A single compartment circuit illustration

The governing system of equations relating the variables $R, L$, and $C$ for this model results from the Kirchhoff current and voltage laws (corresponding to the momentum and mass conservation principles here) applied to a single compartment assumed being filled with an incompressible Newtonian fluid. They take the form [32-34]

$$
\begin{aligned}
\frac{d P_{\text {out }}}{d t} & =\frac{q_{\text {in }}-q_{\text {out }}}{C} \\
\frac{d q_{\text {in }}}{d t} & =\frac{P_{\text {in }}-P_{\text {out }}-R q_{\text {in }}}{L},
\end{aligned}
$$

where for each artery, $P_{\text {in }}, P_{\text {out }}, q_{\text {in }}$, and $q_{\text {out }}$ are inlet blood pressure, outlet blood pressure, inlet flow rate, and outlet flow rate, respectively. For arteries with a radius $r<0.2 \mathrm{~cm}$, the inertial effect is ignored [36], and in this case, the flow rate is given by $q_{\text {in }}=\left(P_{\text {in }}-P_{\text {out }}\right) / R$.

To calculate the parameters $R, L$, and $C$ of each compartment, the following equations [35] are used : Hagen-Poiseuille equation for resistance, $R=8 \mu l / \pi r^{4}, L=\rho l / \pi r^{2}$, and $C=3 \pi r^{3} l / 2 E h$, 
where $r, l, \mu, \rho, h$, and $E$ are the radius of the artery, the length of the arterial segment, the blood viscosity, the blood density, the arterial wall thickness, and the Young modulus, respectively.

Each arterial segment of the full network including the $\mathrm{CoW}$ is represented by a reduced-order OD model containing the three elements $R, L$, and $C$ (see Figures 3C,D, where each of the arterial segments is modeled with a single compartment). In this way, the full network is represented by a distributed lumped parameter model in which multiple lumped compartments are interconnected.

At the bifurcations, the enforcement of the mass conservation principle and of the continuity equation for pressure permits to prescribe the boundary conditions. In order to include the effect of the downstream vasculature, the blood flow model is coupled to the three-element Windkessel model (WK3-lumped parameter model) $[12,37]$ at the outlet of each terminal compartment. In the WK3 model, the equation relating the instantaneous blood pressure and the flow rate reads as follows:

$$
\frac{d p(t)}{d t}+\frac{p}{R_{D} C}=R_{P} \frac{d q(t)}{d t}+\frac{q R_{T}}{R_{D} C}
$$

where $p, q, C, R_{P}$, and $R_{D}$ are the instantaneous pressure at the inlet of the WK3 model, the instantaneous flow rate, the compliance, the proximal resistance, and the distal resistance of the vascular beds, respectively. $R_{T}=R_{P}+R_{D}$ expresses the total peripheral resistance. The sensitivity of the present approach with respect to the choice of the boundary conditions will be discussed in Section 5.3.

It is not easy to solve the first order differential equations 3 and 4 in view of the stiffness of the system. That is why the Fortran version of an implicit numerical integration solver DVODE [38, 39], available on http://www.radford.edu/ thompson/vodef $90 \mathrm{web} /$, is used to solve the system.

\subsection{Problem Specification}

With the physical model above adopted, the general optimization problem presented in Section 2 can be more specified. The optimization parameter $\mathbf{x}$ of size $n$ are the hemodynamic parameters for each segment plus the number of parameters in the termination boundary conditions. The vector $\mathbf{z}$ of size $m$ contains the parameters used to describe the flow rate from the heart. Thus, $m$ corresponds 
either to the number of points in a discrete representation or to the number of parameters for a reduced-order representation of the signal as shown in Section 5.2. The variable $\mathbf{y}_{\text {obs }}$ (called observations) is flow rate time series from MRA\&MRI acquisitions at given arteries as described in Section 4. With time-series observations $\mathbf{y}_{\mathbf{o b s}}(t)$, different functional $J$ can be considered. Following Lal and colleagues[25, 26], this work aims at minimizing a time-dependent functional based on instantaneous incoming information:

$$
J\left(t, \mathbf{y}(\mathbf{x}, \mathbf{z}, t), \mathbf{y}_{\mathbf{o b s}}(t)\right)=\left\|\mathbf{y}(\mathbf{x}, \mathbf{z}, t)-\mathbf{y}_{\mathbf{o b s}}(t)\right\|_{*}=\frac{1}{2}\left\|\mathbf{y}(\mathbf{x}, \mathbf{z}, t)-\mathbf{y}_{\mathbf{o b s}}(t)\right\|^{2} .
$$

\section{PATIENT-SPECIFIC CLINICAL DATA}

This section presents patient-specific clinical data used in the remainder of the paper. These data have been provided by the Department of Neuroradiology of the Centre Hospitalier Régional Universitaire de Montpellier (CHRU), Montpellier, France, and have been extensively described in Lal [26] together with their acquisition procedures.

As a reminder, before and after image acquisition, arterial systolic brachial pressure (SBP) and diastolic brachial pressure (DBP) at rest were measured using a brachial automatic sphygmomanometer (Maglife, Schiller Medical). The pressure values measured were (115 and $72 \mathrm{mmHg}$ ) in the left brachial artery and $(125$ and $72 \mathrm{mmHg})$ in the right one. The ascending aorta and the internal carotid arteries (right and left ICAs) have been considered for the analysis of blood flow rates.

Figure 2 shows two pairs of the magnitude and phase contrast images acquired - one for the ascending aorta (Figure 2B) and the other one for ICAs ( Figure 2C) - and their corresponding blood flow rates (Figure 2A,C). These flow rates have been obtained from the GyroTools software, called GTFlow (http://www.gyrotools.com/products/gt-flow.html/ ) . 
Figure 3 shows the typical 3D model (and morphology) of CoW (see Figure 3A,B) determined through segmentation of a 3D time of flight magnetic resonance angiography (3D-TOF-MRA) of the patient's CoW.

Using these different images, a complex arterial network of 33 arteries (Figure 3d) consisting of the aorta, vertebral, carotid, and brachial arteries together with an integral CoW adapted from Alastruey et al. [10] has been modeled, and as shown in Table I, some geometric measurements such as lengths and radii of CoW's blood vessel have been obtained. More precisely, in the cerebral regions with best/high image quality, lengths and radii of cerebral arteries have been manually extracted from MRA using RadiANT DICOM Viewer software (http://www . radiantviewer.com/ ), while in the regions with poor image quality, they were obtained from average data reported in the literature. Also, other geometries, such as the carotid vascular tree one, could not be obtained because their acquisition requires the injection of contrast material called gadolinium, which is impossible to perform on healthy volunteers. To fill this kind of geometries and all the missing geometry of the full network, data reported in the literature were also necessary. 
Table I. Geometric parameters corresponding to arterial segments (and compartments) in Figure 3 measured from magnetic resonance imaging. The missing geometry (marked with an asterisk) of larger arteries is taken up from the average data in the literature [10,12]. $\mathrm{R}$ indicates right and $\mathrm{L}$, left.

\begin{tabular}{|c|c|c|c|c|c|c|c|}
\hline Id & Name & $l, \mathrm{~cm}$ & $r, \mathrm{~cm}$ & Id & Name & $l, \mathrm{~cm}$ & $r, \mathrm{~cm}$ \\
\hline 1 & Ascending aorta (AA) & $4.00^{*}$ & $1.200^{*}$ & 18 & L. post. comm. artery (L.PCoA) & 1.20 & 0.075 \\
\hline 3 & Aortic $\operatorname{arch} \mathrm{I}(\mathrm{Aa} \mathrm{I})$ & $2.00 *$ & $1.120^{*}$ & 20 & R. post. comm. artery (R.PCoA) & 1.20 & 0.075 \\
\hline 5 & R. common carotid (R.CC) & $17.7^{*}$ & $0.250^{*}$ & 22 & R. external carotid (R.ECA) & $17.7^{*}$ & $0.150^{*}$ \\
\hline 6 & L. common carotid (L.CC) & $20.8^{*}$ & $0.250^{*}$ & 23 & L. internal carotid I (L-ICA) & $17.7^{*}$ & 0.200 \\
\hline 7 & Aortic arch II (Aa II) & $3.90^{*}$ & $1.070^{*}$ & 24 & L. external carotid (L.ECA) & $17.7^{*}$ & $0.150^{*}$ \\
\hline 10 & R. brachial (R.BRA) & $42.2^{*}$ & $0.403^{*}$ & 27 & L. middle cerebral artery (L.MCA) & 11.9 & 0.143 \\
\hline 11 & R. vertebral (R.VA) & $14.8^{*}$ & $0.136^{*}$ & 28 & L. anterior cerebral artery A1 (L.ACA, A1) & 1.20 & 0.117 \\
\hline 12 & L. vertebral (L.VA) & $14.8^{*}$ & $0.136^{*}$ & 29 & R.middle cerebral artery (R.MCA) & 11.9 & 0.143 \\
\hline 13 & L. brachial (L.BRA) & $42.2^{*}$ & $0.403^{*}$ & 30 & R. anterior cerebral artery A 1 (R.ACA, A1) & 1.20 & 0.117 \\
\hline 14 & Basilar (BAS) & 2.70 & 0.150 & 31 & R. anterior cerebral artery A2 (R.ACA, A2) & 10.3 & 0.120 \\
\hline 15 & R. post. cerebral artery P1 (R.PCA, P1) & 0.56 & 0.110 & 32 & Anterior comm. artery (ACoA) & 0.30 & 0.074 \\
\hline
\end{tabular}

The inverse hemodynamic problem aims at identifying unknown parameters (the arterial stiffness and the WK3 model boundary parameters) for the network as shown in Figure 3D and as described in Lal and colleagues [25, 26]. In the parameter estimation problem, both available patient-specific flow rate waveforms for the right internal carotid (R-ICA; \#21 in Table I) and the left internal carotid (L-ICA; \#23 in Table I) were used as observations during EnKF assimilation steps. Blood rheological parameters $\mu$ and $\rho$ were set at $0.004 \mathrm{~Pa} \mathrm{~s}$ and $1050 \mathrm{~kg} \mathrm{~m}^{-3}$ and at the inlet (ascending aorta, compartment \#1 in Figure 3D), specific values of flow rates, $q_{i n}$, measured by PC-MRI (see Figure 2A,B) were imposed.

The results shown in the next section were obtained under the following assumptions on the unknown model parameters: 
- The parameters $R, L, C$, and the WK3 boundary condition parameters are assumed to well reproduce the patient-specific description of the OD blood flow model expressed by Equation 3.

- Eh, the product of Young modulus and thickness of arteries, is the unknown quantity to recover by data assimilation, and it is assumed to be given by this empirical formula [40]:

$$
E h=r\left(k_{1} e^{k_{2} r}+k_{3}\right)
$$

where the radius $(r)$ is measured from MRA. An estimation of the product $E h$ is found by looking for an estimation of the unknown constants $k_{i}$ with their initial guesses as $k_{1}=2.0 \times 10^{7} \mathrm{~g} \mathrm{~cm}^{-1} \mathrm{~s}^{-2}, k_{2}=-22.0 \mathrm{~cm}^{-1}$ and $k_{3}=8.0 \times 10^{5} \mathrm{~g} \mathrm{~cm}^{-1} \mathrm{~s}^{-2}$.

- For each left and right pair of terminal compartments, the same WK3 parameters are assumed by symmetry. For instance, the terminal compartments \#22 and \#24 are assigned with the same WK3 boundary conditions. The parameters $R_{P_{i}}, R_{D_{i}}$ and $C_{i}$ where $i=9,10,19,22,29,31$ denotes the compartment numbers are also considered as unknown model parameters. Thus, 21 parameters consisting of six proximal resistances, six distal resistances, six compliances, and three constants defining the product $E h$ are estimated. The initial estimates for the proximal resistances and the compliances were taken from the data published by Alastruey et al.[10], and the initial guesses for the distal resistances $R_{D}$ are chosen such that the ratio $R_{P} / R_{T}=0.2$, ie, $R_{D}=4 R_{P}[41]$. 


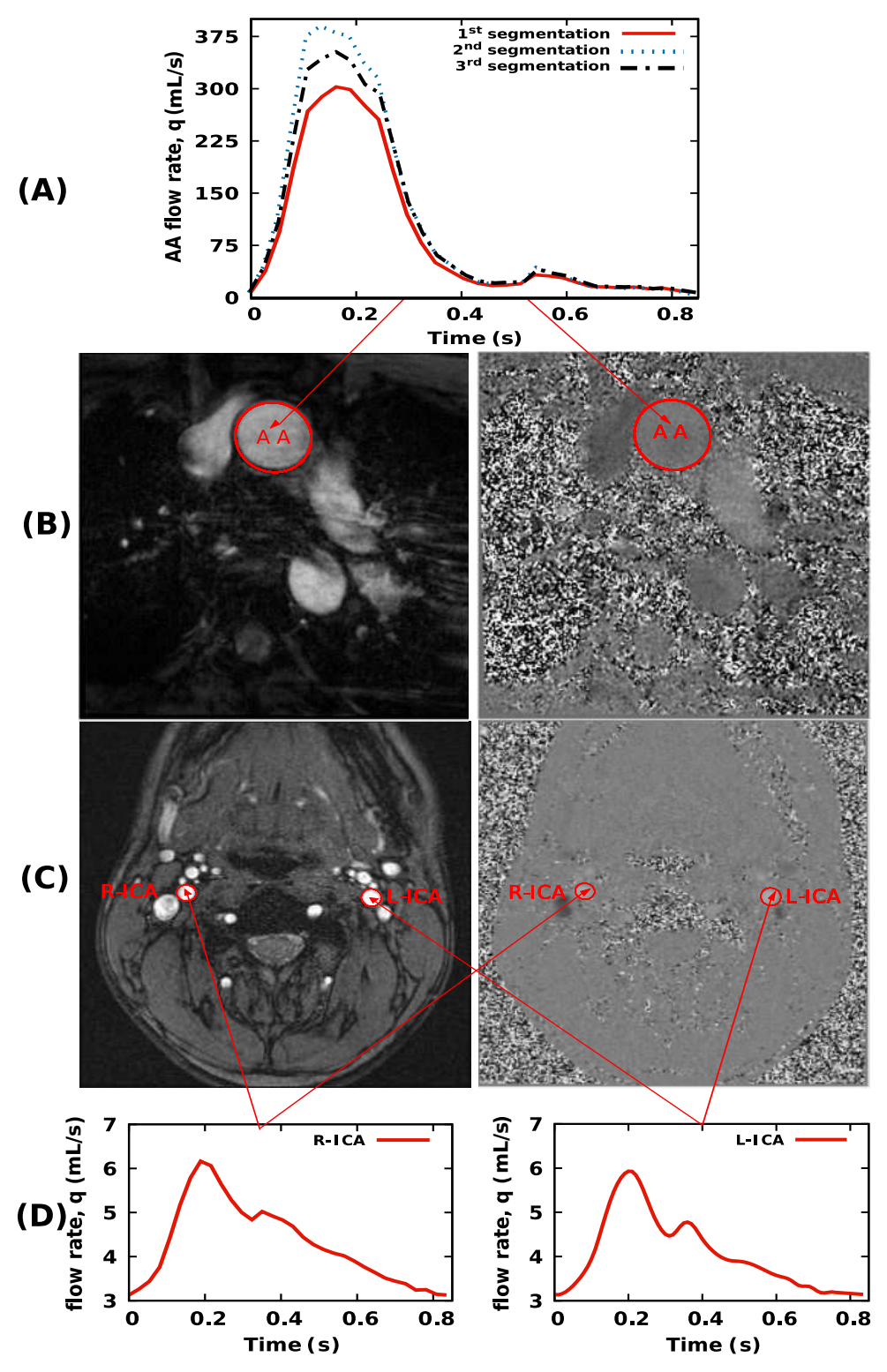

Figure 2. PC-MRI of the patient-specific ascending aorta and internal carotid arteries (right and left) showing the blood flow through one of the selected slices. In the center, on the left are the magnitude images and on the right are the phase contrast images $\mathrm{B}$, and $\mathrm{C}$, with Venc setting of 200 and $80 \mathrm{~cm} \mathrm{~s}^{-1}$ for the ascending aorta and the internal carotid arteries, respectively. The instantaneous blood flow rate values, $q(t)$ are acquired at each time frame and are plotted against time for one cardiac cycle as shown on the top for AA A, and at the bottom for ICAs D. In panel A, the signals obtained from three different segmentations performed by the same "operator" are reported to give a feeling of the uncertainty on the inlet flow rate. 

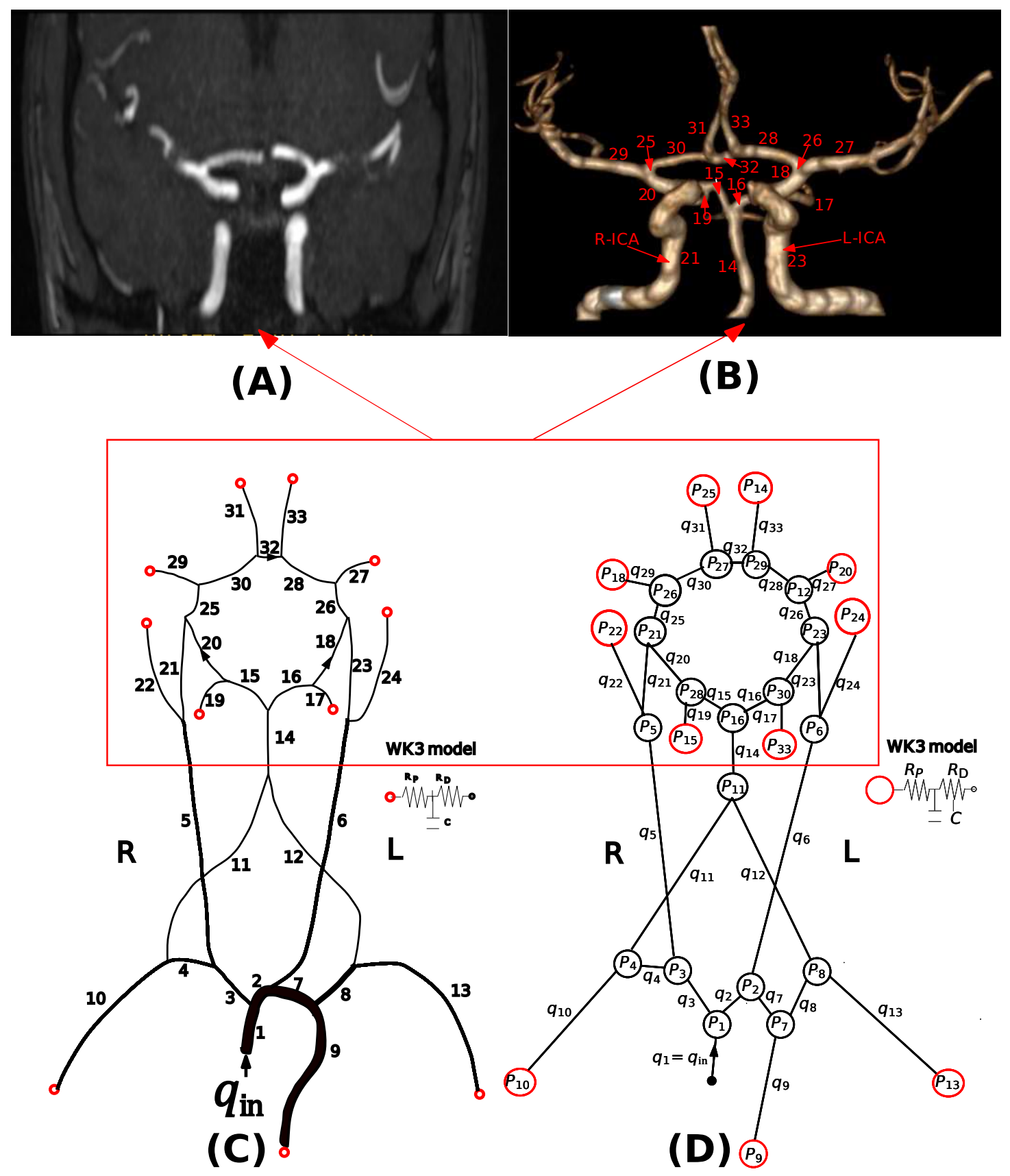

Figure 3. A TOF MRI scan A, and the corresponding segmented 3D model of the CoW B, for the considered patient-specific case. The network [10] of a 1D blood flow model of the upper body arteries and of the CoW $\mathrm{C}$, and its equivalent compartment model $\mathrm{D}$. The lines size indicates relative size of the arteries. The numbers on segmented model refer to the Ids of the arterial segments in Table I. Arrows indicate the direction of flow. Flow rates are assigned the compartment numbers corresponding to those in Table I. At the inlet (ascending aorta, compartment \#1), specific values of flow rates, $q_{i n}$, are imposed. 


\section{PATIENT-SPECIFIC RESULTS}

This section presents typical convergence of the inversion algorithm and sensitivity analysis for the inverse problem with respect to several modeling issues and parameters of the direct model. Before presenting these results, it seems appropriate to clarify first the meaning of convergence in the remaining of this paper.

More precisely, it is said that there is convergence if, on the basis of a visual inspection, the estimated parameters have only very minor variations from systole to diastole on the one hand, and on the other, from one cardiac cycle to the next. Quantitative parameters for convergence could be introduced, but it would weigh down the procedure.

\subsection{Convergence}

Figure 4 shows the target and model signals in time. One observes that there is a good agreement between the target and predicted flow rate waveforms after 10 cycles (about $8.35 \mathrm{~s}$ ).

Figures 5 and 6 show the evolutions of the first two moments of the 21 estimated optimization parameters $\mathrm{x}$. One sees very different convergence patterns. Some variables do not fully converge even though the observations are well recovered. This suggests that those variables have small effects on the solution. The final estimates of the 21 parameters with their associated uncertainties are tabulated in Table II. As the most important information is the elastic characteristics of the vessels, figure 7 shows the evolutions of three of the estimated Young moduli during the EnKF iterations. As the Young moduli are obtained through a same expression, they bare similar convergence patterns. The most important thing here to note is that all the Young moduli for the 33 arteries tend to realistic values with respect to the literature (ie, between 0.1 and $1.2 \mathrm{MPa}$ ) $[10,42,43]$. 

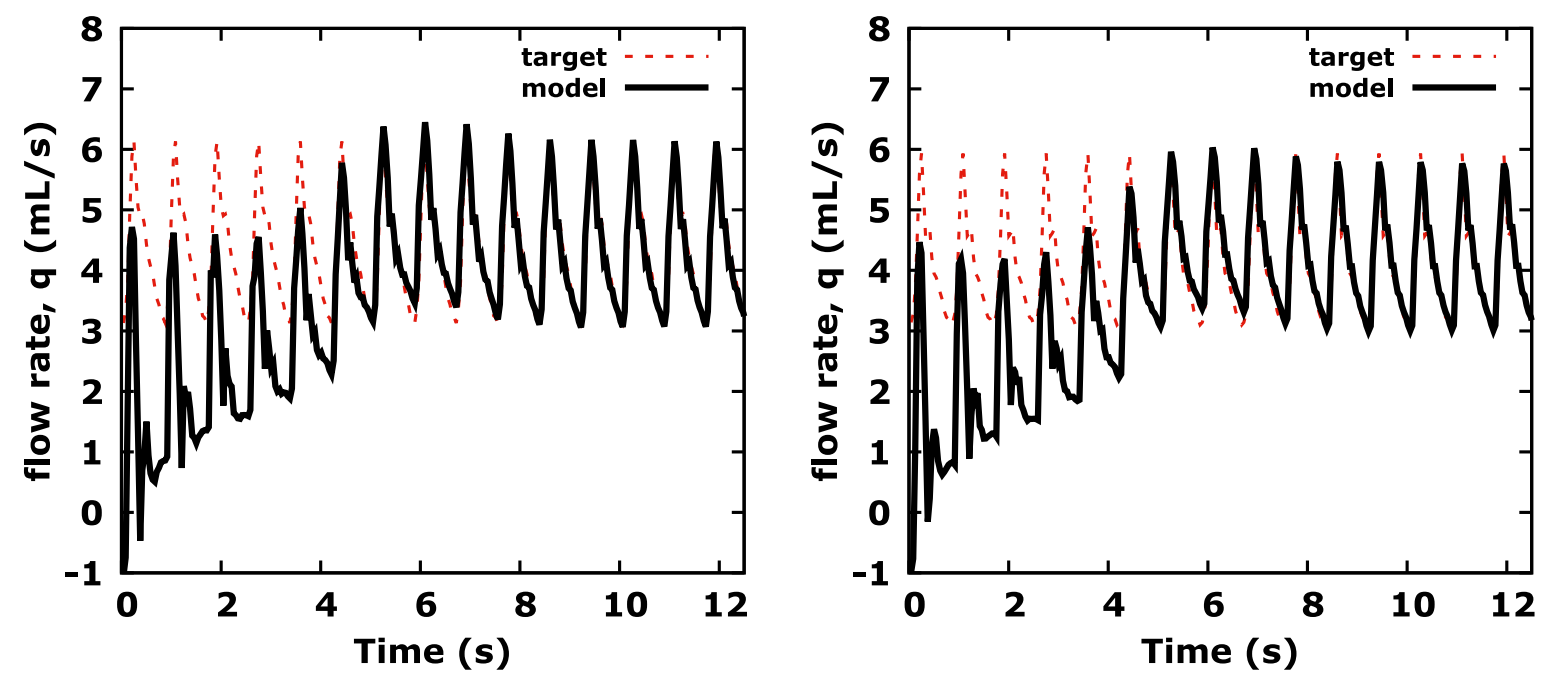

Figure 4. Comparison of the model simulated blood flow rate waveform in R-ICA (on the left) and L-ICA (on the right) to the target signals.
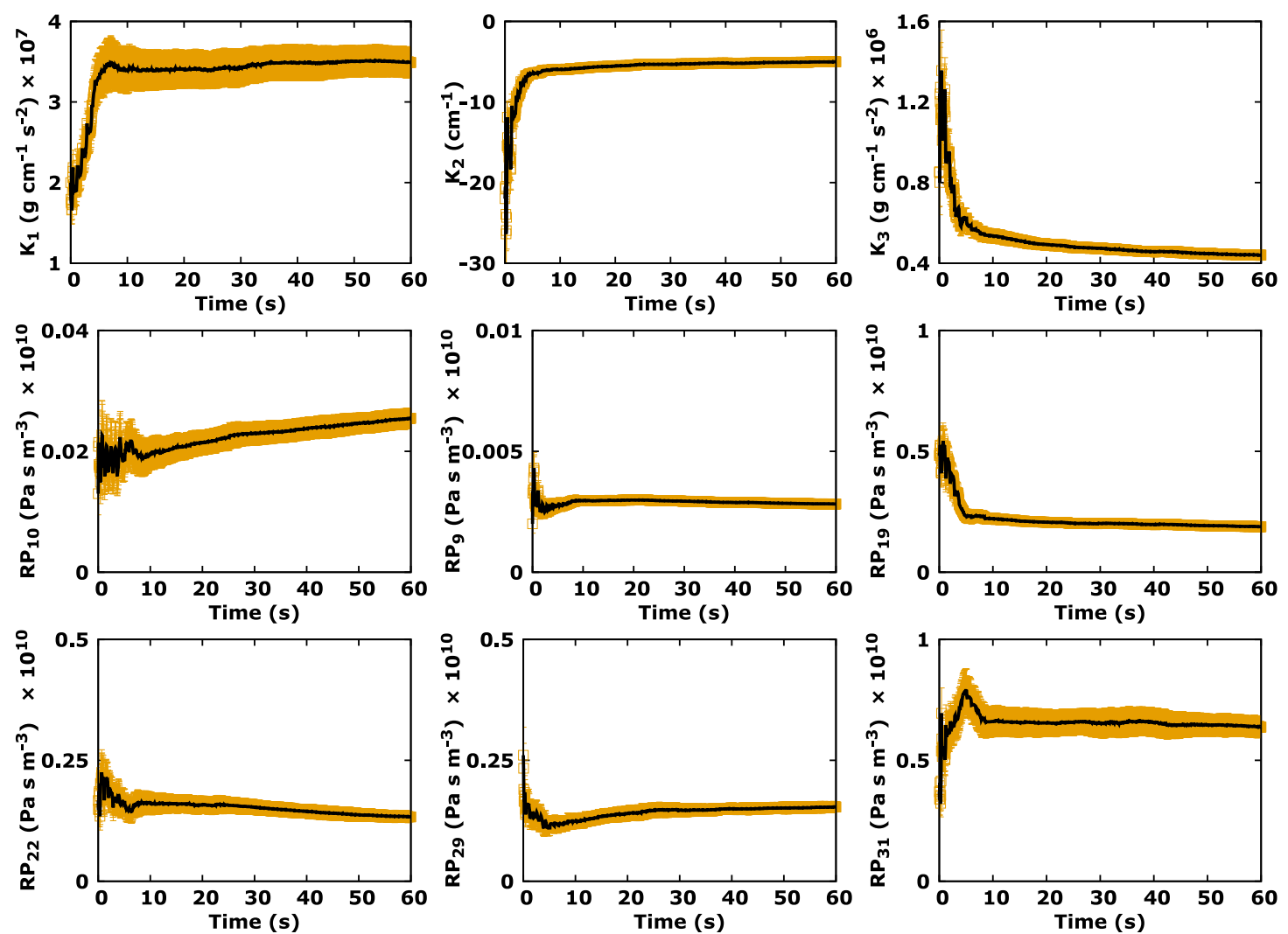

Figure 5. Histories of the mean and standard deviation of the nine first estimated parameters during EnKF iterations. 

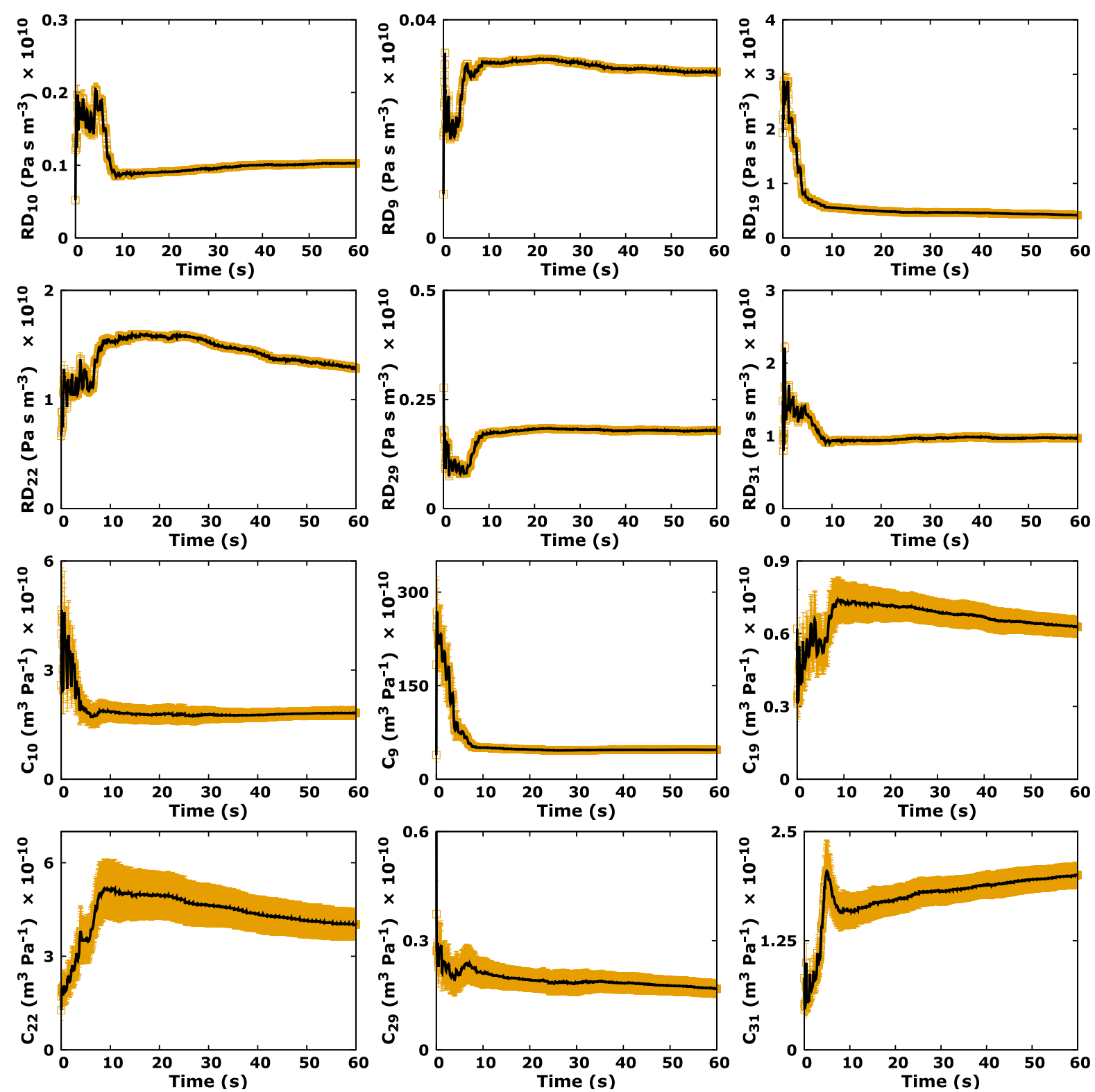

Figure 6. Histories of the mean and standard deviation of the 12 last estimated parameters during EnKF iterations. 
Table II. Estimated parameters with the associated errors for the patient-specific. The values of constants $k_{1}, k_{2}$, and $k_{3}$ are in $\times 10^{7} \mathrm{~g} \mathrm{~cm}^{-1} \mathrm{~s}^{-2}, \mathrm{~cm}^{-1}$, and $\times 10^{5} \mathrm{~g} \mathrm{~cm}^{-1} \mathrm{~s}^{-2}$ respectively. The proximal $\left(R_{P}\right)$ and distal $\left(R_{D}\right)$ resistances are in $\times 10^{9} \mathrm{~Pa} \mathrm{~s} \mathrm{~m}^{-3}$ and the compliance $(C)$ are in $\times 10^{-10} \mathrm{~m}^{3} \mathrm{~Pa}^{-1}$.

\begin{tabular}{|lcc|}
\hline Parameter & Initial guess & EnKF estimate \pm error \\
\hline$k_{1}$ & 2.00 & $3.49 \pm 0.19$ \\
$k_{2}$ & -22.0 & $-5.00 \pm 0.26$ \\
$k_{3}$ & 8.5 & $4.39 \pm 0.24$ \\
$R_{P 9}$ & 0.02 & $0.028 \pm 0.001$ \\
\hline$R_{P 10}$ & 0.13 & $0.25 \pm 0.017$ \\
\hline$R_{P 19}$ & 4.8 & $1.88 \pm 0.15$ \\
\hline$R_{P 22}$ & 1.67 & $1.33 \pm 0.12$ \\
\hline$R_{P 29}$ & 2.61 & $1.54 \pm 0.11$ \\
\hline$R_{P 31}$ & 3.70 & $6.39 \pm 0.43$ \\
\hline$R_{D 9}$ & 0.08 & $0.30 \pm 0.0005$ \\
\hline$R_{D 10}$ & 0.52 & $1.03 \pm 0.007$ \\
\hline$R_{D 19}$ & 19.32 & $4.19 \pm 0.034$ \\
\hline$R_{D 22}$ & 6.68 & $12.88 \pm 0.174$ \\
\hline$R_{D 29}$ & 10.44 & $1.79 \pm 0.018$ \\
\hline$R_{D 31}$ & 14.80 & $9.69 \pm 0.068$ \\
\hline$C_{9}$ & 38.78 & $47.12 \pm 2.62$ \\
\hline$C_{10}$ & 2.58 & $1.82 \pm 0.17$ \\
\hline$C_{19}$ & 0.62 & $0.63 \pm 0.043$ \\
\hline$C_{22}$ & 1.27 & $4.02 \pm 0.49$ \\
\hline$C_{29}$ & 1.16 & $0.17 \pm 0.023$ \\
\hline$C_{31}$ & 0.82 & $2.00 \pm 0.15$ \\
\hline & & \\
\hline
\end{tabular}




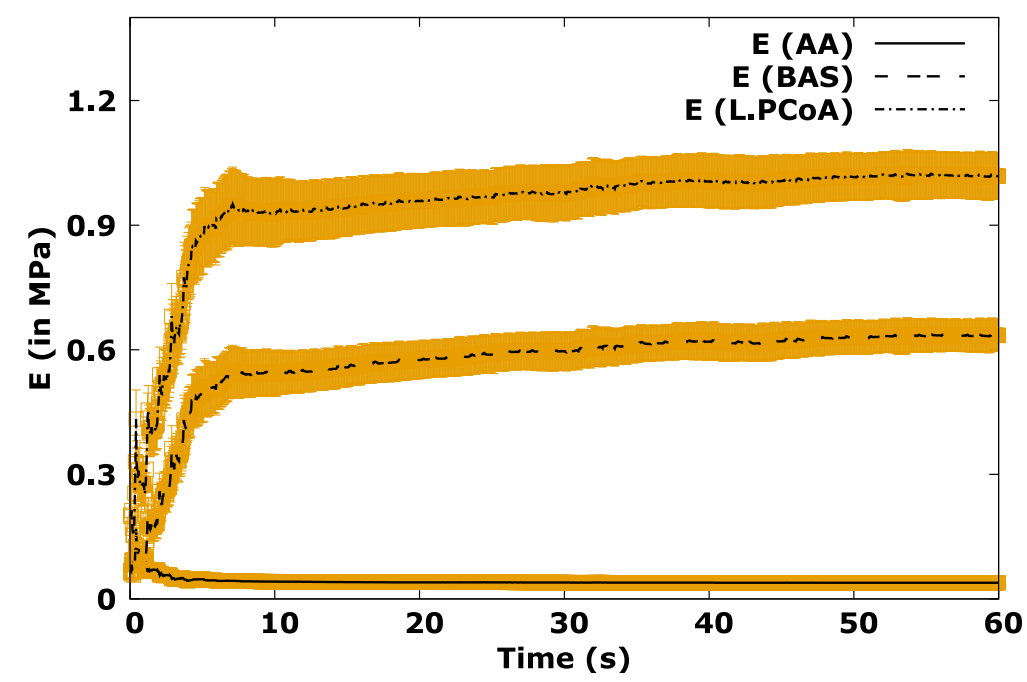

Figure 7. Mean and standard deviation of estimated Young moduli for three of the 33 segments of the network. The evolution is over $8.35 \mathrm{~s}$ corresponding to about 10 cardiac cycles.

To illustrate this, a compared overview of some of estimated parameters with those reported in previous studies [10, 12, 41, 43-45] was carried out, and the model-simulated flow rates through all the termination models and carotid arteries were also examined. The results of the comparison of the parameters are shown in Figure 8 and the model-simulated flow one in Figures 4 and 9. These latter are also compared with blood flow rates values reported in Alastruey et al [10] and Reymond et al [12] and with clinically measured blood flow rates using MRI in Table III. One notices that almost all estimated parameters were found to be in the same order of magnitude than those reported in other studies [10, 12, 41, 43-45]. Furthermore, one observes good overall agreement in flow waveforms but considerable differences in flow amplitude at all arterial terminations. All mean, systolic, and diastolic flow rates reported in Alastruey et al [10] and Reymond et al [12] are much higher than model outputs except those of MCA. This is to a large extent due to the difference of patients and models considered in investigated cases and especially to flow rate difference from the beginning in the ascending aorta $\left(420,-33\right.$, and $96 \mathrm{~mL} \mathrm{~s}^{-1}$ for systolic, diastolic, and mean flow rate, respectively, in Reymond et al [12] versus 302, 0, and $62.4 \mathrm{~mL} \mathrm{~s}^{-1}$ for systolic, diastolic, and mean flow rate, respectively, in the model). This prevents from properly comparing the different flow divisions of the model with those reported in Alastruey et al [10] and Reymond et al [12]. 
Despite these substantial differences, note that the model provided flow rate predictions that faithfully reproduced wave characteristics in the ICAs. This is illustrated in Figure 4, which shows the comparison between the target (clinically measured blood flow rates in the ICAs using MRI) and blood flow model simulations (predictions) based on the estimated parameters. From the results, the comparison between the assimilated OD model and in vivo data (MRI) is fair. All mean, systolic, and diastolic flow rates measurements using MRI and model outputs in ICAs differ by less than $5 \%$, and thus, one can conclude, in the absence of other clinically measured flow rates, that the $0 \mathrm{D}$ model considered here may very well predict blood flow rate in the entire arterial tree.
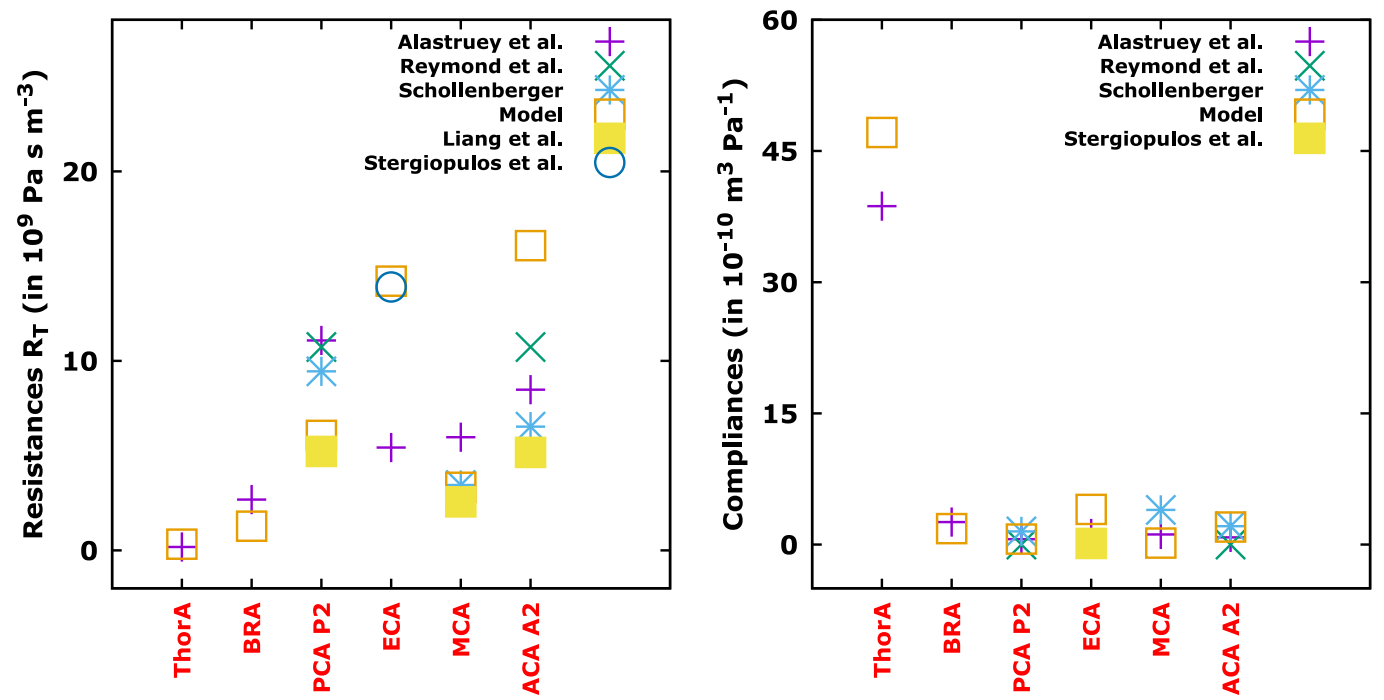

Figure 8. Comparison of estimated compliances and total peripheral resistances at the arterial terminations with reports in the literature. The estimated parameters using the assimilated compartment model (represented by empty squares) are in the same order of magnitude than those existing in the literature. 

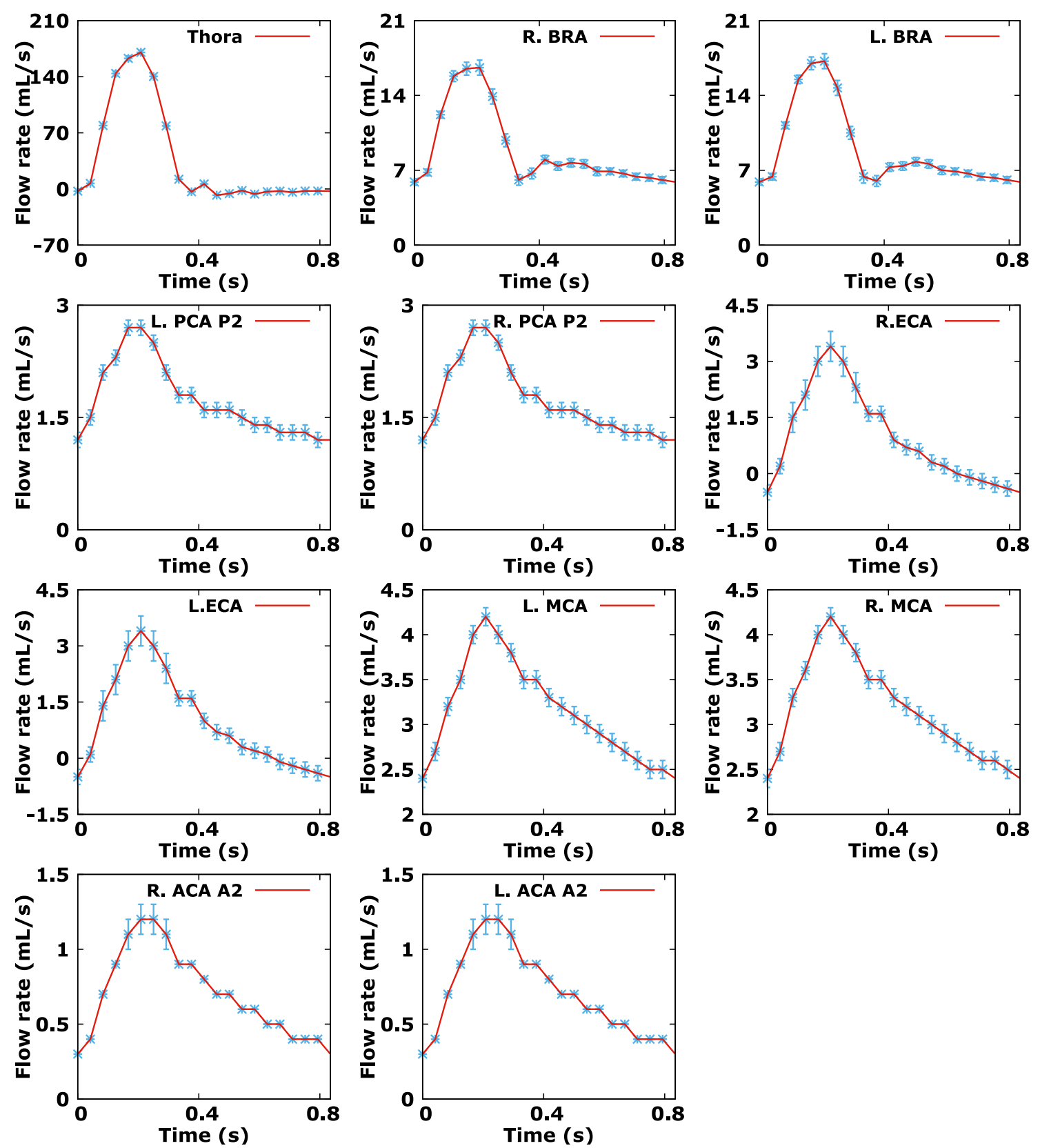

Figure 9. Mean and standard deviation of estimated blood flow rate at the arterial terminations. 
R. RAPADAMNABA $E T A L$ 


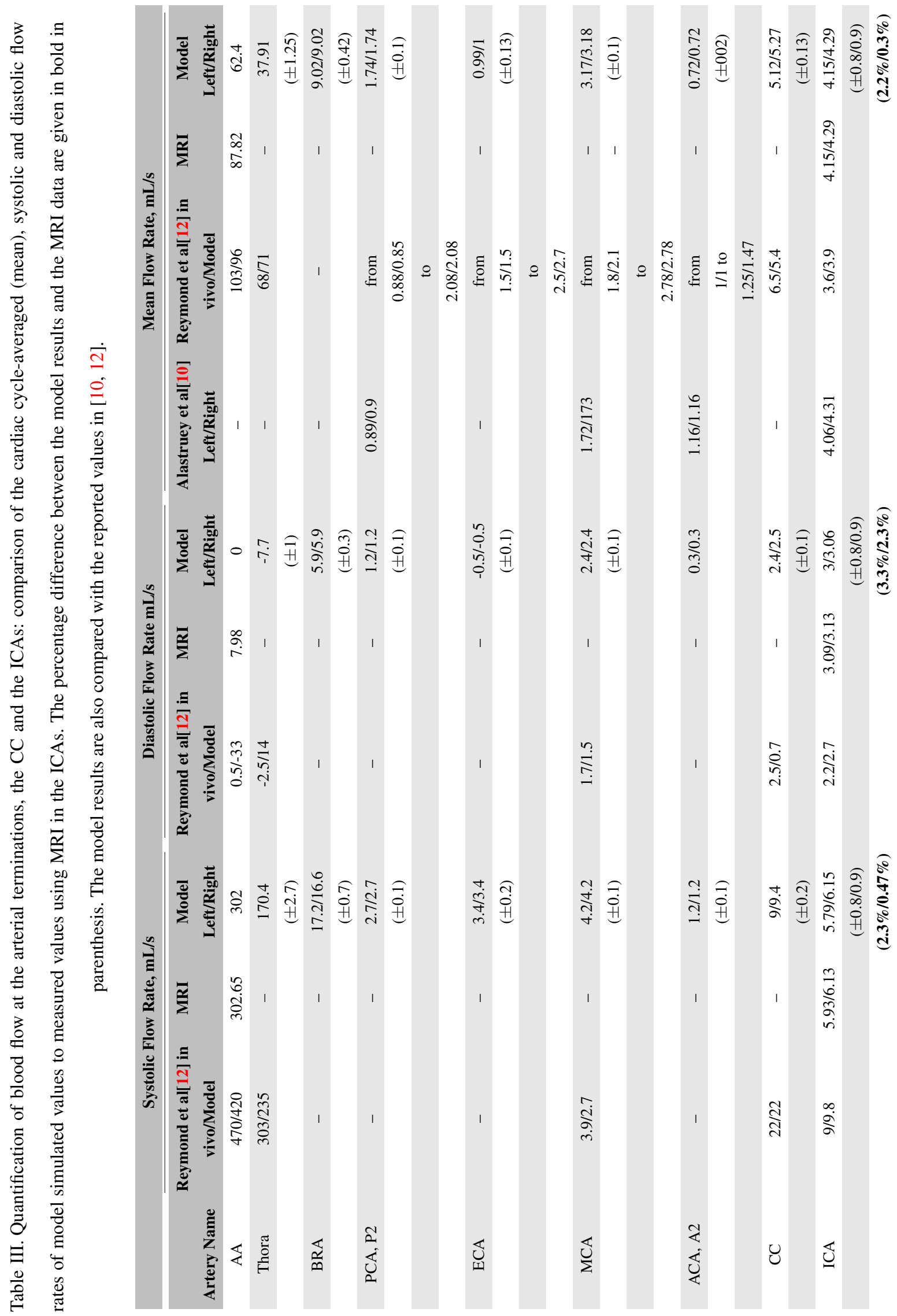




\subsection{Sensitivity analysis with respect to the inlet flow rate $q_{i n}(t)$}

One important source of uncertainty in the inversion is due to the variability in the segmentation procedure by the "operator" in charge from PC-MRI data to define the variable called $\mathrm{z}$ in the minimization problem (1).

Let us consider $q_{i n}(t)$ the patient-specific blood flow rate time series through one of the selected slices in the ascending aorta as shown in Figure 2A for one cardiac cycle. As presented in this figure, $q_{i n}(t)$ is defined in a deterministic way. One however observes that different segmentations, even by a same "operator", lead to different flow rates time series. To analyze the sensitivity of the inversion with respect to this uncertainty, it is convenient to represent $q_{i n}(t)$ using a reduced-order model. For that purpose, the model proposed in Alastruey et al [10] involving two parameters which will be considered as stochastic here (see Figure 10) will be adapted as follows:

$$
q_{i n}(t)= \begin{cases}\alpha \sin (\pi t / \tau) & \text { if } t<\tau \\ \varepsilon & \text { otherwise }\end{cases}
$$

where $\alpha\left(\mathrm{mL} \mathrm{s}^{-1}\right)=\mathcal{N}\left(\mu_{\alpha}, \sigma_{\alpha}^{2}\right), \tau(\mathrm{s})=\mathcal{N}\left(\mu_{\tau}, \sigma_{\tau}^{2}\right)$, and $\varepsilon\left(\mathrm{mL} \mathrm{s}^{-1}\right)=\mathcal{N}\left(\mu_{\varepsilon}, \sigma_{\varepsilon}^{2}\right)$ are the components of $\mathbf{z}$ in (1). $\mu_{\alpha}, \mu_{\tau}$, and $\mu_{\varepsilon}$ are patient-specific. They are chosen solving a least square problem to fit the inflow rate shown in figure 2. This leads to the following values: $\mu_{\alpha}=302 \mathrm{~mL}$ $\mathrm{s}^{-1}, \sigma_{\alpha}=15 \mathrm{~mL} \mathrm{~s}^{-1}, \mu_{\tau}=0.35 \mathrm{~s}, \sigma_{\tau}=0.0175 \mathrm{~s}, \mu_{\varepsilon}=21.65 \mathrm{~mL} \mathrm{~s}^{-1}$, and $\sigma_{\varepsilon}=1.0825 \mathrm{~mL} \mathrm{~s}^{-1}$. The standard deviations that are of the order of $5 \%$ of mean values model the uncertainty that an "operator" will introduce through the manual acquisition/segmentation steps from dicom format files. 


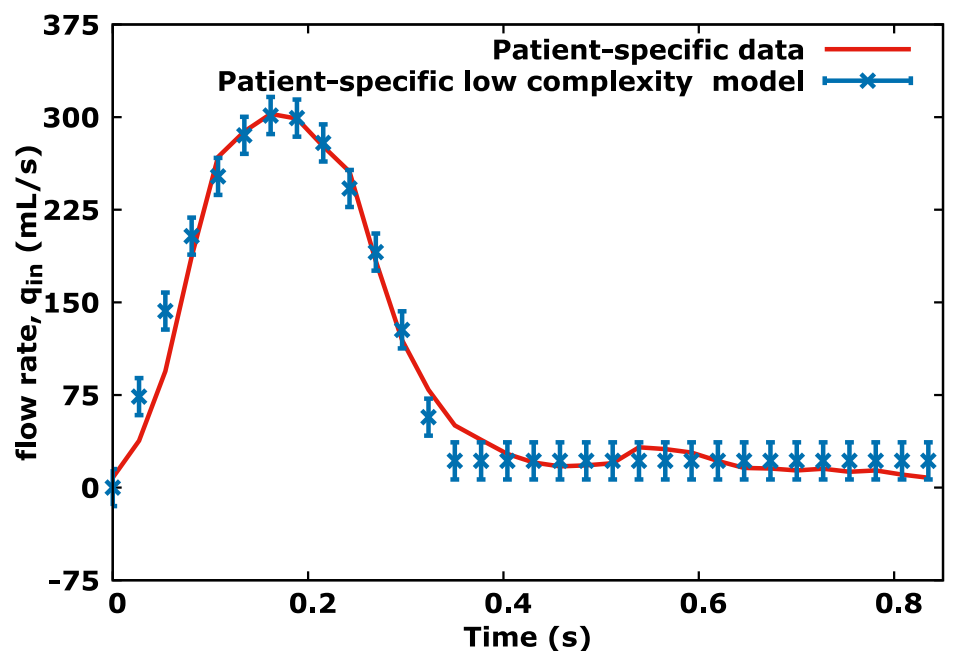

Figure 10. Uncertain inflow rate $q_{i n}$ and the low complexity model. The latter will be used for uncertainty propagation in inversion.

To analyze the sensitivity of the inversion with respect to the uncertainty on $q_{i n}(t), 30$ inversions for Gaussian sampling in $\alpha, \tau$, and $\varepsilon$ have been performed. An ensemble of size 30 has been used for all inversions. This ensemble size choice is based on a synthetic case study results that have shown that an ensemble of size 30 seemed to be good enough to estimate 21 model parameters for the prediction of cerebral arterial pressure with an error of less than $10 \%$ [26]. To generate the 30 ensemble members, one proceeds as follows: from initial estimate of $n$ unknown parameters (mean $\bar{x}_{l}$ and variance $P_{l}$ for $l=1, \ldots, n$ ), we randomly initialize an ensemble of parameters, $\mathbf{x}^{i}$, for $i=1 \ldots, q_{\mathrm{ens}}$ where $\mathbf{x}^{i}=\left(x_{1}, x_{2}, \ldots, x_{n}\right)$ and $x_{l} \sim \mathcal{N}\left(\bar{x}_{l}, P_{l}\right)$ for $l=1, \ldots, n$. For more details, the reader is referred to Lal [26]. It is interesting to illustrate the convergence not for the optimization variables $\mathrm{x}$ but for the Young moduli, which are obtained from following expression (5): $E=r\left(k_{1} e^{k_{2} r}+k_{3}\right) / h$ where $k_{1}, k_{2}$, and $k_{3}$ are the three first components of $\mathbf{x}$.

Once all the inversions are completed, one can proceed with some statistics. Figure 11 shows the estimated mean value and standard deviation for the Young modulus of each segment. One sees that the "operator" uncertainty can have up to $5 \%$ impact on the elastic parameter estimation. This is therefore of the order of the patient-specific uncertainty in $\mathbf{z}=(\alpha, \tau, \varepsilon)$ in equation 6 and obtained assimilating uncertain patient data by the low complexity $q_{i n}$ model. This means that the procedure 
seems to work linearly in connection with the errors propagation. This is not an aberration as a result; on the contrary, this is satisfactory in that it reveals that the procedure does not seem to reduce nor to amplify the errors. However, $q_{i n}$ needs to receive very accurate treatment if one would like to have an uncertainty below say $5 \%$ on the outcomes, which is may be difficult to achieve given the nature of the problem and also the way data are acquired.

Figure 12 presents the estimated mean and standard deviation for the right brachial pressure. It is quite reassuring to notice that despite the "operator" uncertainty, the predicted brachial pressure remains coherent with the ausculatory measure of the patient using a sphygmomanometer (ie, between 72 and $125 \mathrm{mmHg}$ ) taken after the acquisition. This gives some indication for the level of uncertainty one can tolerate because of manual acquisition steps.

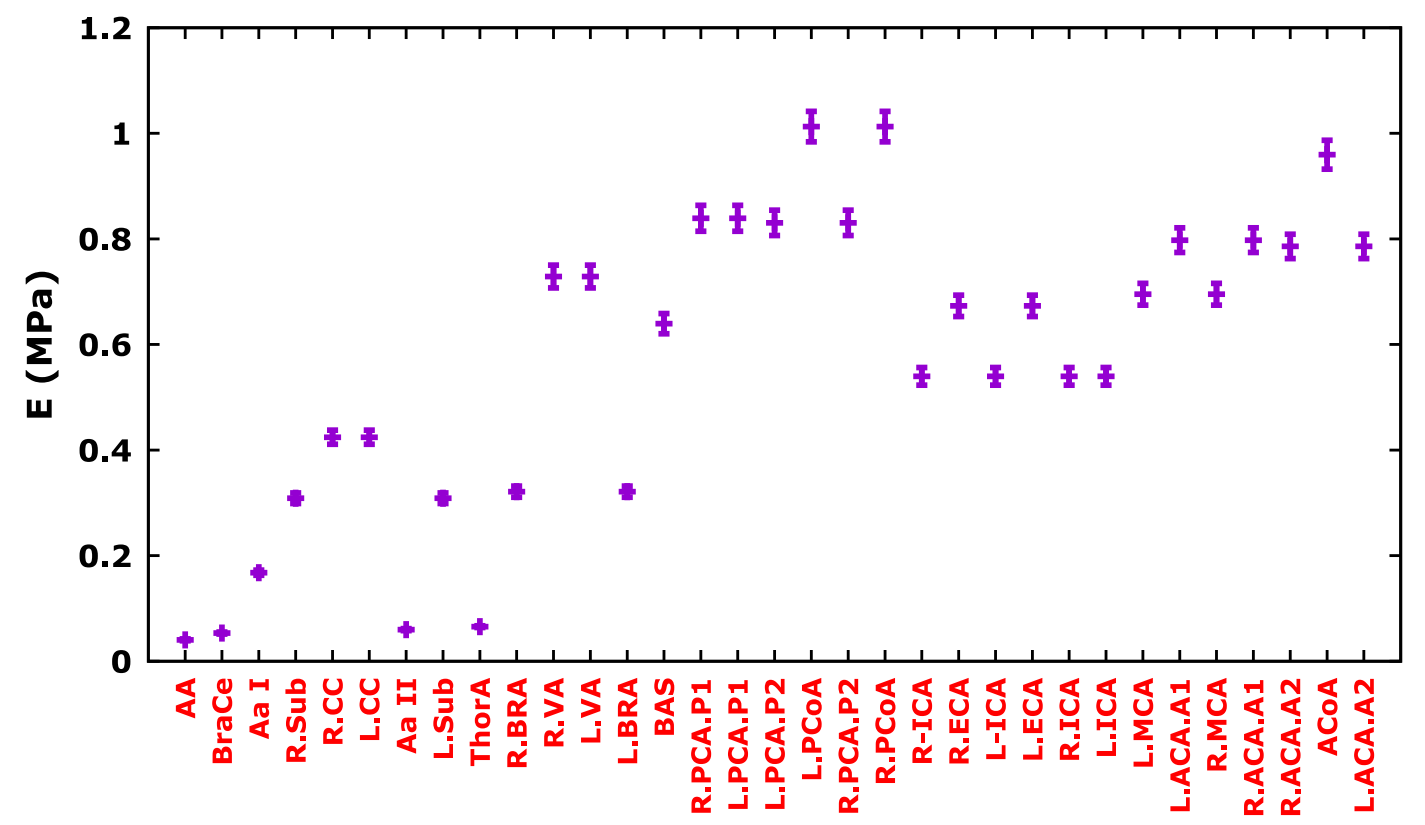

Figure 11. Mean and standard deviation of different segments Young moduli showing the impact of the uncertainty on the inlet flow rate $q_{i n}(t)$ on the inversion. 


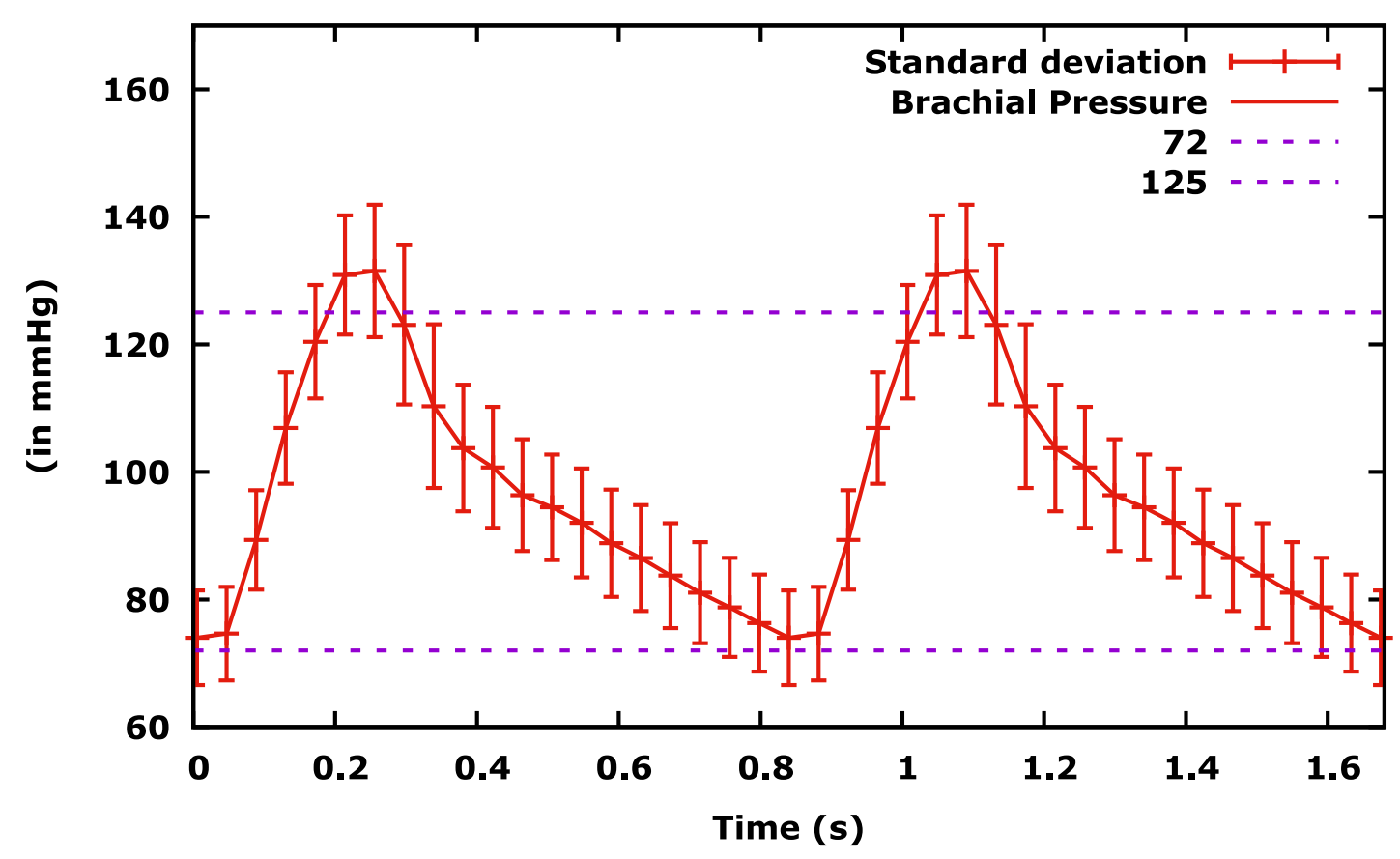

Figure 12. Right brachial pressure mean and standard deviation for two cardiac cycles showing the impact of the uncertainty on $q_{i n}(t)$ on the inversion. The clinically measured SBP and DBP are 125 and 72 , respectively.

\subsection{Sensitivity analysis with respect to the boundary conditions}

Another important source of uncertainty in the inversion is related to the choice of the boundary conditions at the outlet of each of the terminal compartments. As described in Section 3.1, the blood flow model uses a three-element Windkessel condition, which couples the instantaneous blood flow rate and pressure through equation 4 . It would be interesting to see how the use of a simpler boundary condition will affect the inversion. More precisely, WK1 boundary conditions where the blood flow rates at terminations are related to the instantaneous pressure through a simple algebraic relation involving the proximal and distal resistances are considered:

$$
p=q R_{T}=q\left(R_{P}+R_{D}\right)
$$

Hence, a WK1 condition is a WK3 one with zero compliance. One advantage of WK1 is a reduction of the number of optimization variables from 21 to nine: the six total resistances $R_{T_{i}}$ 
with $i=9,10,19,22,29,31$ and the three constants $k_{1}, k_{2}$, and $k_{3}$ necessary for the definition of Eh. However, the same ensemble size of 30 is used for both inversions.

Figures 13,14, and 15 show the impact on the inversion of a change of the boundary conditions from WK3 to WK1. This analysis shows that the WK1 conditions are clearly insufficient to describe the underlying physics of the problem. Indeed, despite the inversion's success in recovering the target flow rate at R-ICA and L-ICA segments, the brachial pressures are miscalculated. One also sees that the convergence of the EnKF algorithm is not fully achieved. Actually, some parameters do not converge at all and this despite the ensemble size advantages in the WK1 inversion. Yet, to be adopted, the prescribed boundary conditions must allow at the same time to provide good convergence for the estimated parameters, to well reproduce both available patient-specific flow rate waveforms for the right internal carotid and the left internal carotid and to well predict brachial blood pressure. If one of these expected results is not achieved by the chosen boundary conditions, this choice cannot be relevant. As a conclusion, only WK3 conditions will be considered for the next sensitivity analysis regarding the interrogations of the assumed symmetry in the terminations boundary conditions.
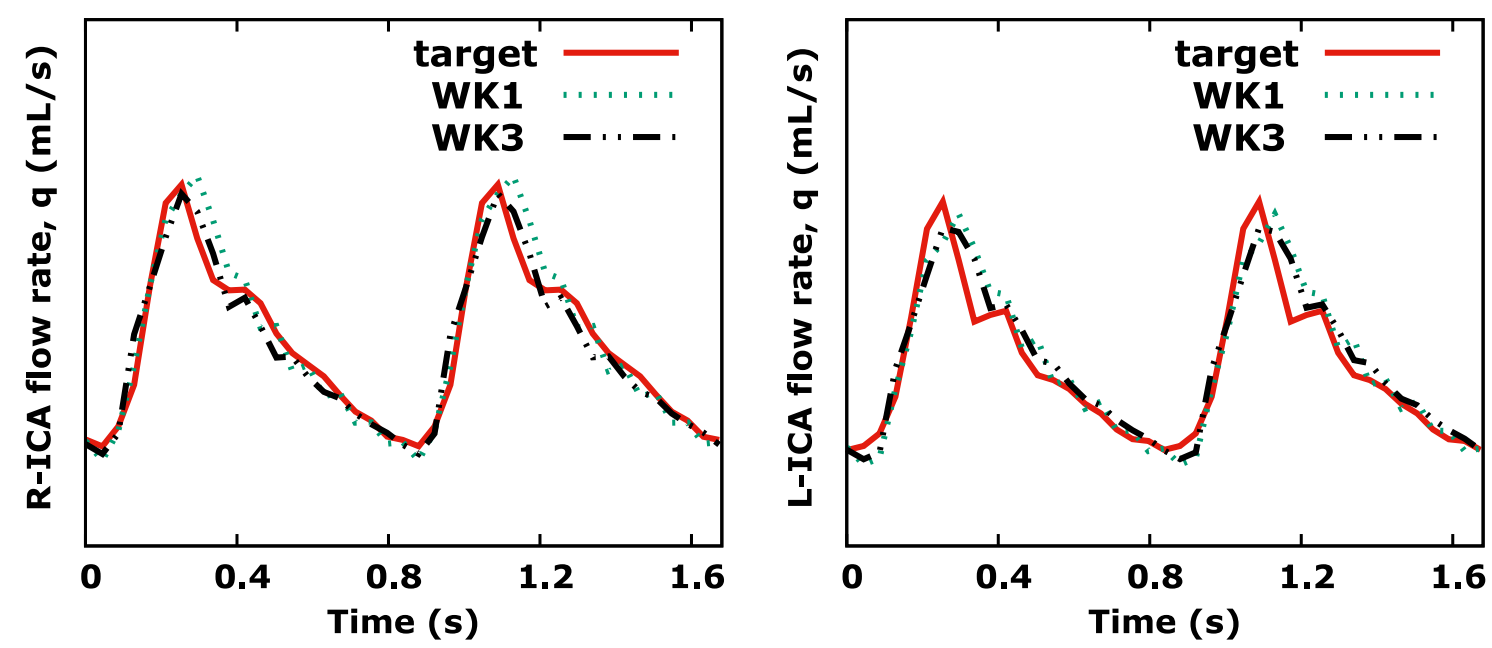

Figure 13. Flow rate waveform using WK1 and WK3 and comparison with the target flow rate in R-ICA and L-ICA. 

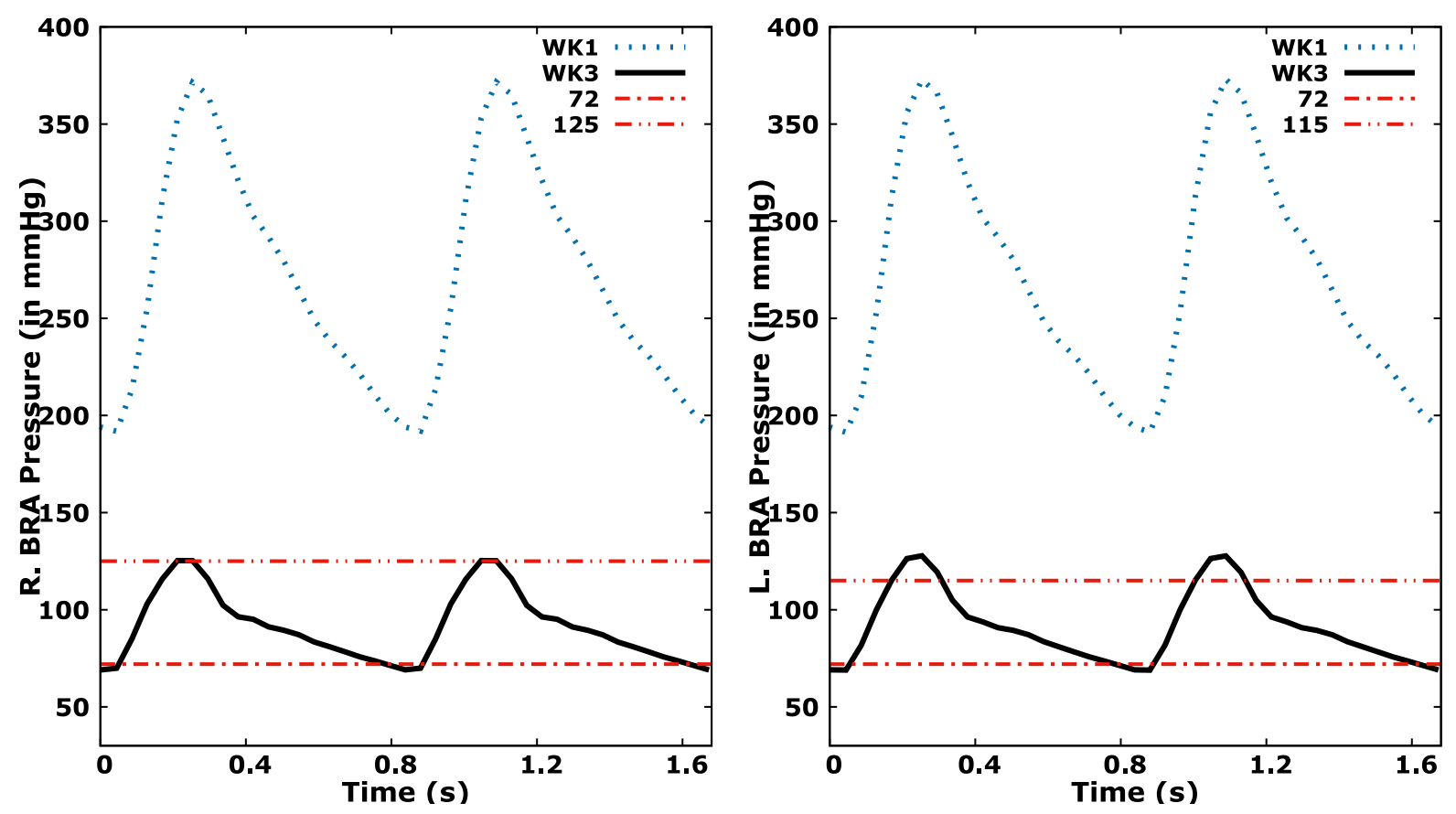

Figure 14. Comparison between R. and L. brachial pressures with WK1 and WK3. The clinically measured SBP and DBP in the right and the left brachial arteries are 125 and 72, and 115 and 72, respectively. 

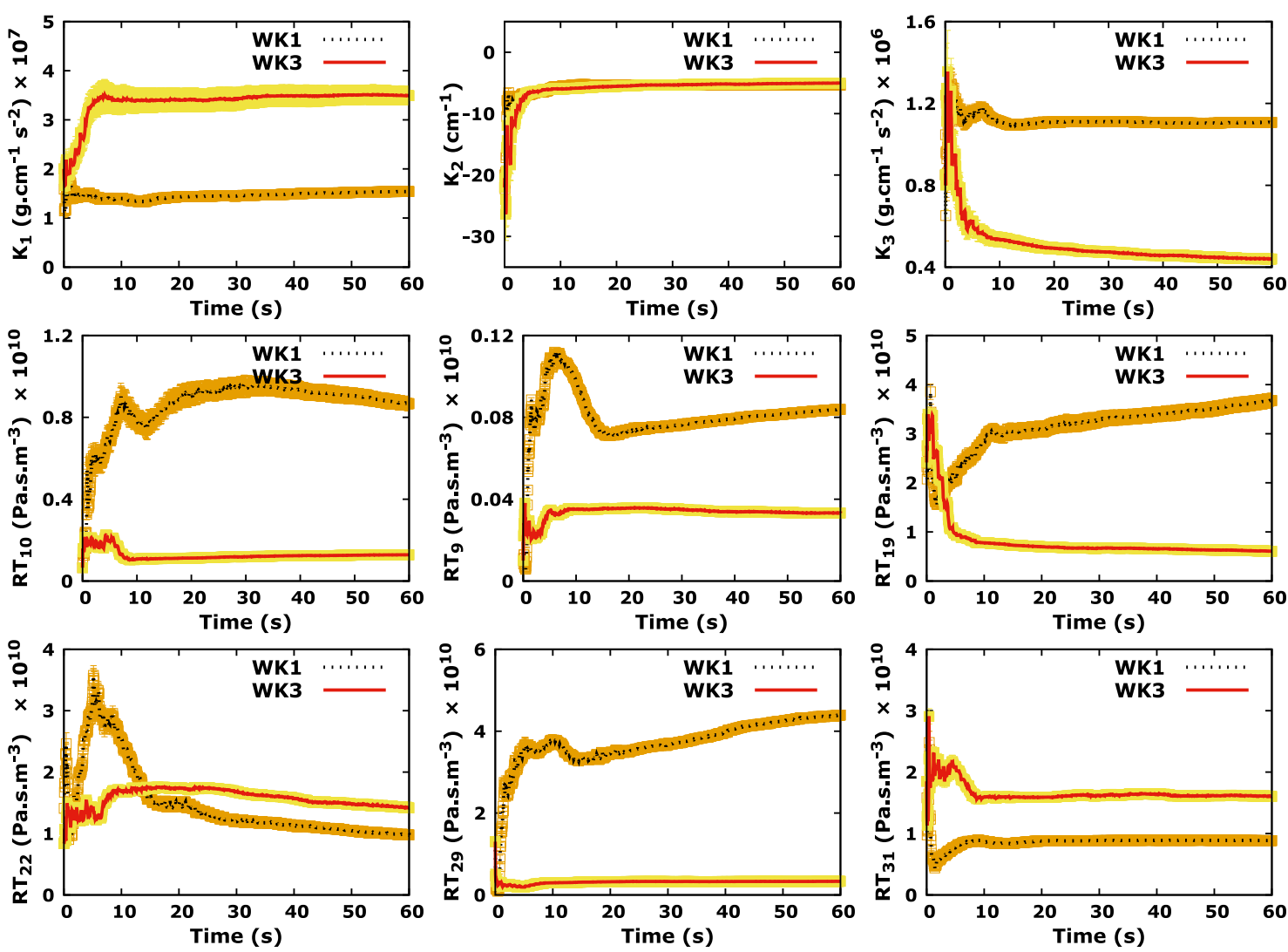

Figure 15. Convergence of estimated parameters during EnKF using WK1 and WK3. The shaded areas represent the standard deviation around the ensemble mean values (dashed and solid lines).

As presented in Section 4, the same WK3 boundary conditions have been applied at the left and right pairs of the terminal compartments. To analyze the sensitivity of the inversion with respect to this assumption, one proceeds with several nonsymmetric scenarios. Unfortunately, to the knowledge of the authors, no indication on how the nonsymmetry takes place can be found in the literature. In other words, it is unclear if, for instance, the termination resistances or compliance are higher or lower on the left with respect to the right-hand side. Actually, it seems that the nonsymmetry is patient-dependent. The analysis will be carried out in three steps: nonsymmetric resistances with symmetric compliances, nonsymmetric compliances with symmetric resistances, and nonsymmetric resistances and compliances.

Let us start analyzing the effect of nonsymmetry in the resistances on the inversion keeping the compliances symmetric. The nonsymmetry has been introduced considering $R_{D}^{l e f t}=a_{R} R_{D}^{\text {right }}$ 
and $R_{P}^{l e f t}=a_{R} R_{P}^{r i g h t}$ where $a_{R}$ is a constant. Several inversions have been made for the values of $a_{R}=0.5,0.75,1$ (symmetric WK3), 1.25 and 1.5, meaning that for each value chosen for $a_{R}$, the parameter identification procedure has been applied using the EnkF algorithm, which leads to the full set of physiological parameters. These values of $a_{R}$ have been a priori chosen, and different sampling can be considered without difficulty. This is an illustration of how the inversion procedure can be used to analyze a possible hypothesis made by practitioners on the possibility of nonsymmetric behavior in terminations. Also, the sampling is made in order for the resistance to have admissible physiological values around the reference value " 1 " corresponding to the symmetric WK3 boundary conditions case. $R_{D}^{\text {left,right }}$ and $R_{P}^{\text {left,right }}$ replace $R_{D}$ and $R_{P}$ in expression (4) in corresponding left and right terminations. Figures 16, 17, and 18 show the impact of this nonsymmetry on the inversion. One notices that a loss of symmetry on the resistances does not impact the flow rate. However, it plays a big role in pressure estimation as the mean brachial pressures and the SBP-DBP differences (DeltaP) increase with the level of resistances on the left terminations.
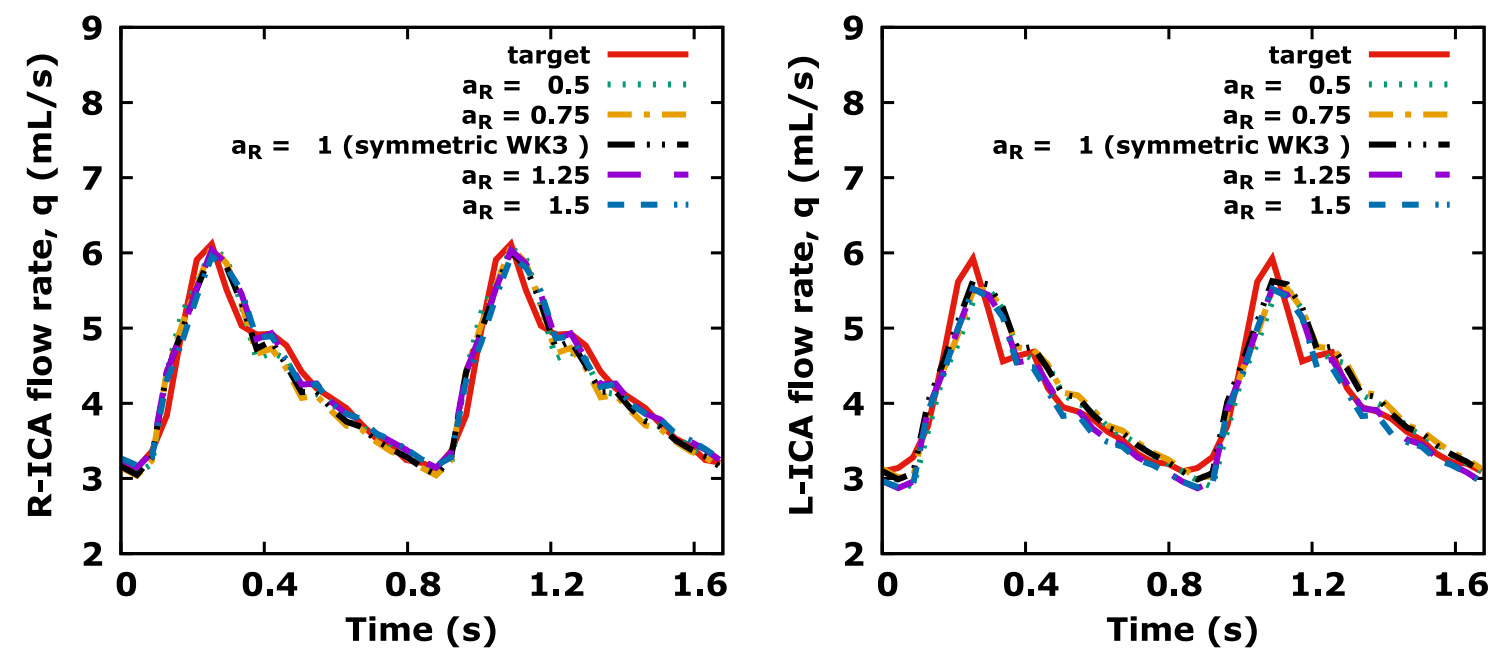

Figure 16. Flow rate waveform using WK3 with the same compliances and different resistances for left and right pairs of terminal compartments and comparison with the target flow rate in ICAs. 

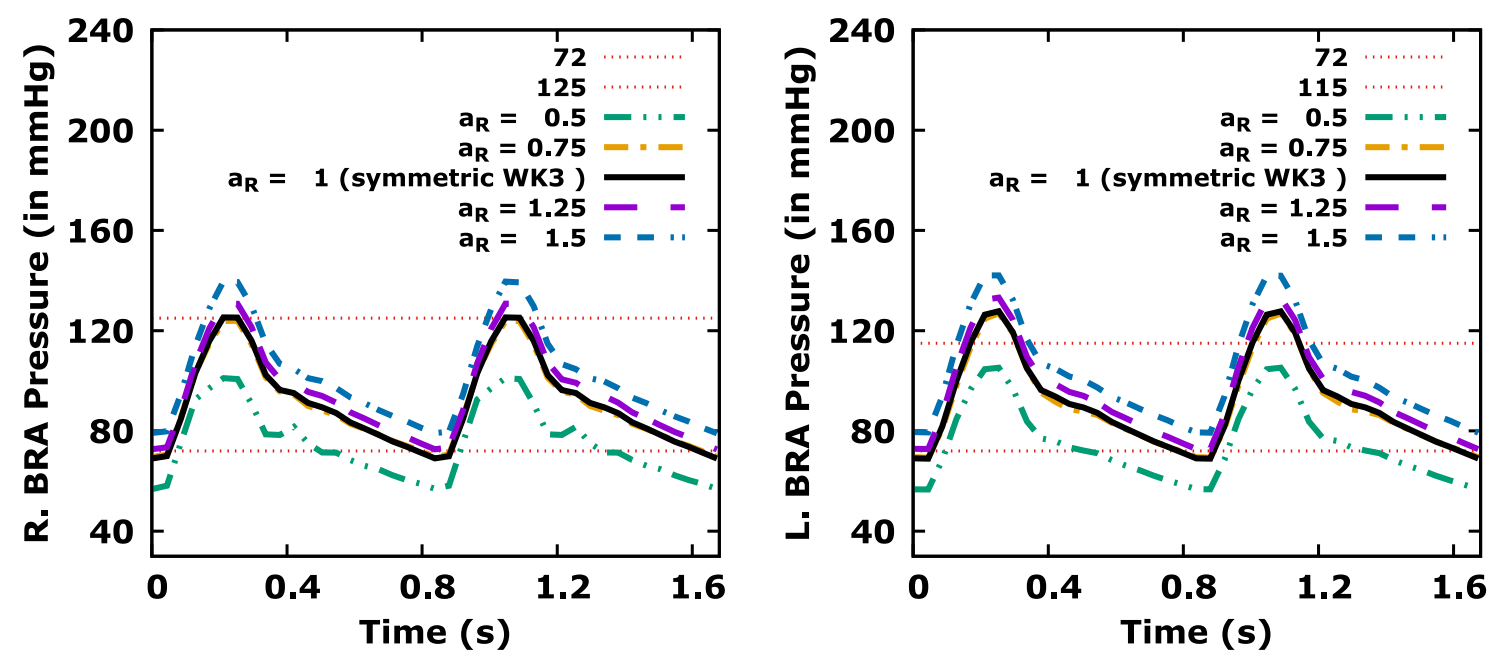

Figure 17. Comparison between R. and L. brachial pressures estimated using WK3 with the same compliances and different resistances for left and right pairs of ICAs' terminal compartments. The clinically measured SBP and DBP in the right and the left brachial artery are 125 and 72, and 115 and 72, respectively.
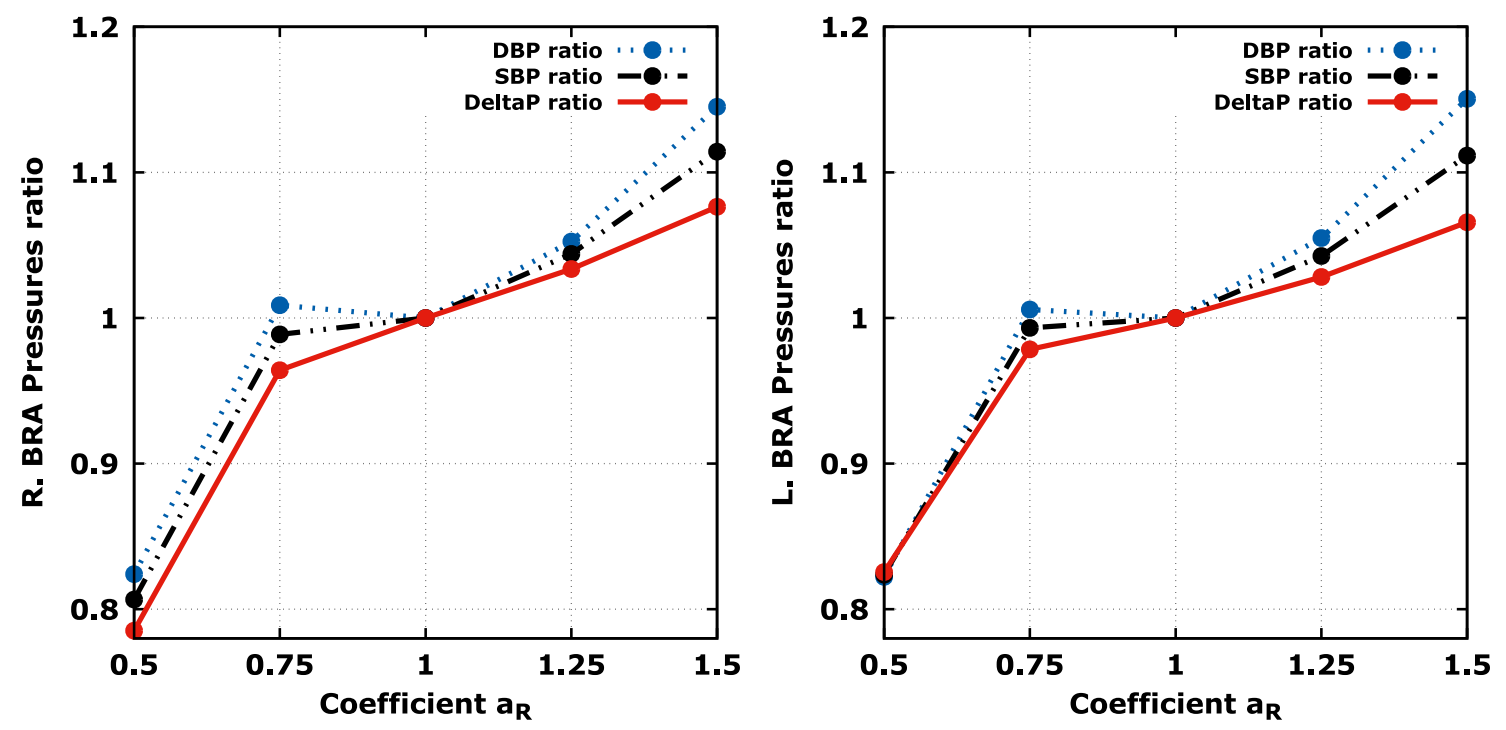

Figure 18. Rate of variation of different pressure information with respect to $a_{R}$. The different pressure information increase with the level of resistances.

Now, let us see the impact of a nonsymmetry on the compliances for symmetric resistances. The nonsymmetry is again introduced through a multiplication by a constant $a_{C}$ on the left compliances, $C^{l e f t}=a_{C} C^{r i g h t}$. Again, several inversions have been performed for the values of $a_{C}=0.5,0.75,1$ (symmetric WK3), 1.25 and 1.5. $C^{\text {left,right }}$ replace $C$ in expression (4) in corresponding left and 
right terminations. Figures 19, 20, and 21 show the impact of this nonsymmetry on the inversion. It appears that, unlike with the nonsymmetry in the resistances, a loss of symmetry on the compliances has much less impact on the inversions.

As a conclusion, nonsymmetry in the resistances seems to play a much bigger role.
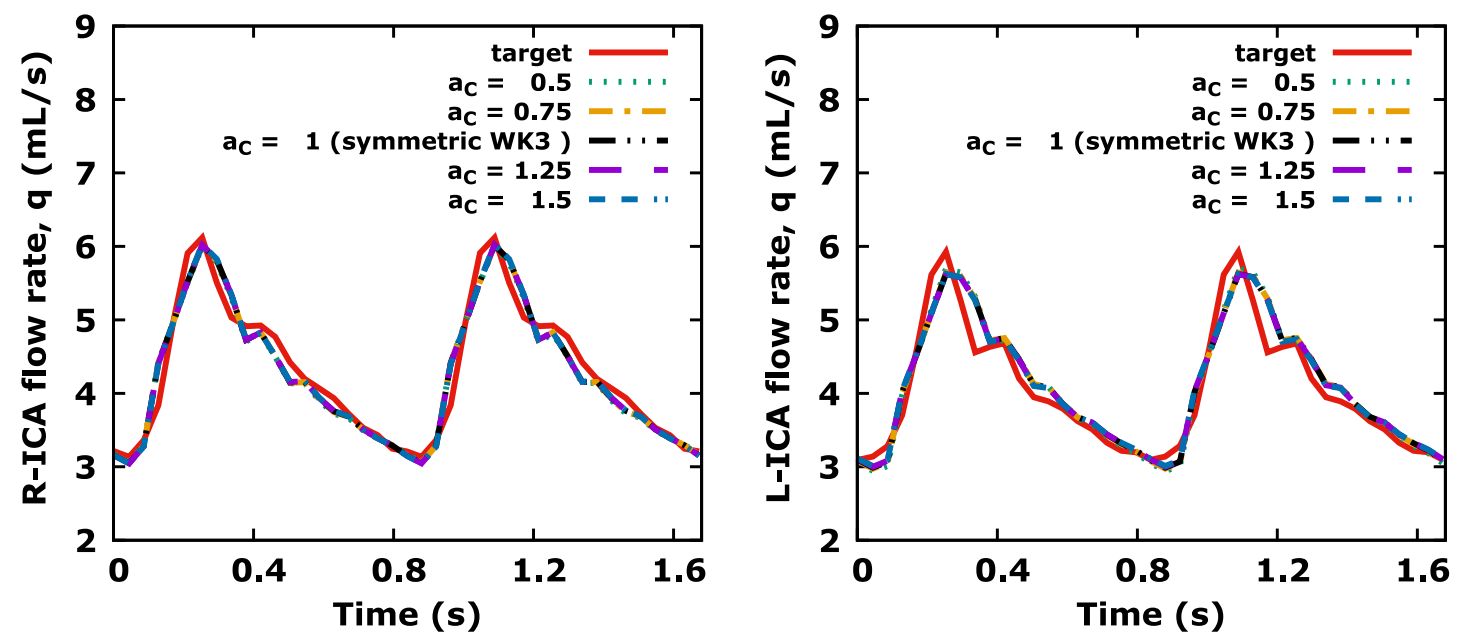

Figure 19. Comparison of the model simulated blood flow rate waveform using WK3 with the same resistances and different compliances for left and right pairs of ICAs' terminal compartments.
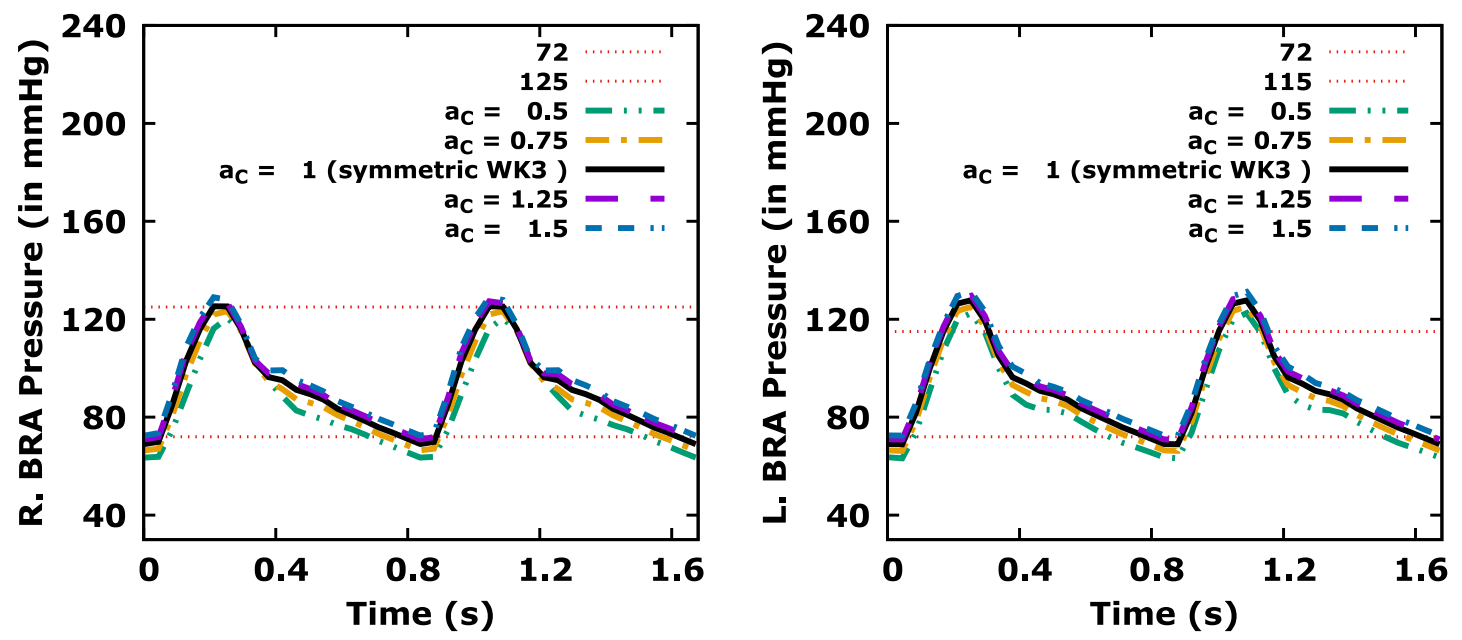

Figure 20. Comparison between R. and L. brachial pressures estimated using WK3 with the same resistances and different compliances for left and right pairs of ICAs' terminal compartments. The clinically measured SBP and DBP in the right and the left brachial artery are 125 and 72, and 115 and 72, respectively. 

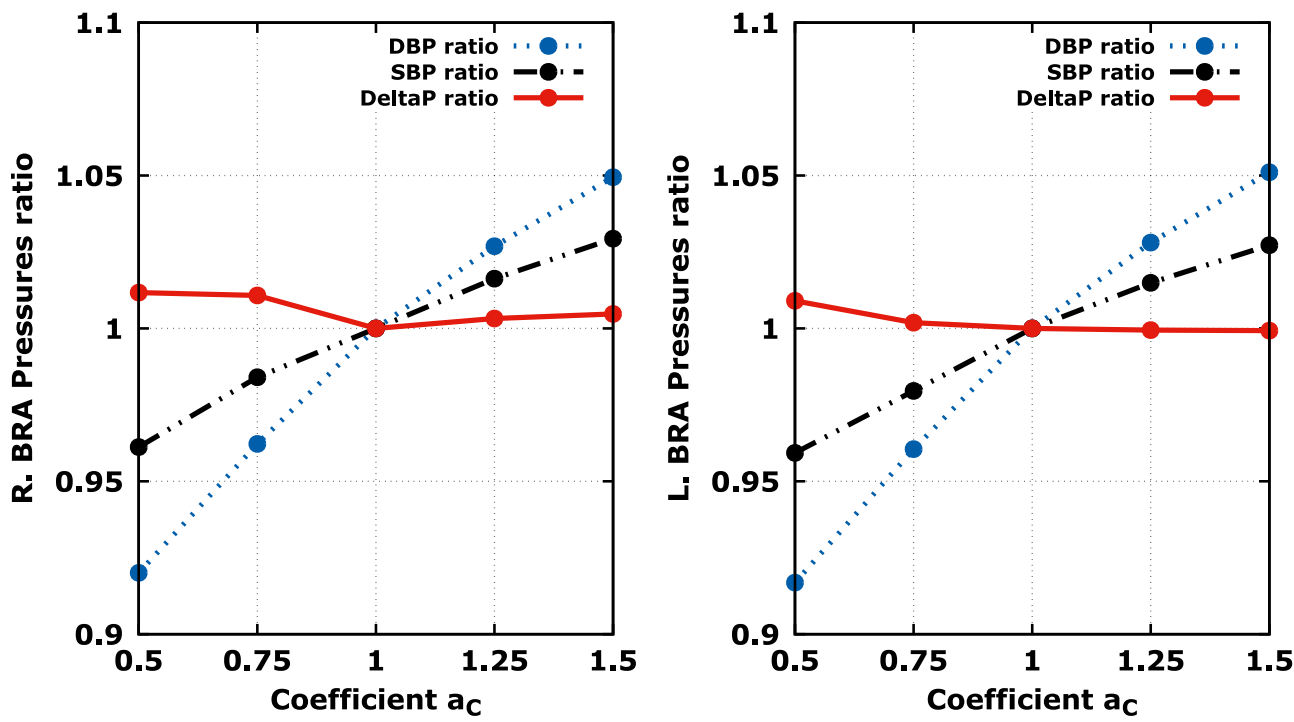

Figure 21. Rate of variation of different pressure information with respect to $a_{C}$. Despite the systolic and diastolic pressures vary, their difference DeltaP is nearly invariant with respect to this nonsymmetry.

Finally, both the resistances and compliances are considered as nonsymmetric. Figures 22, 23, and 24 show the impact of this loss of symmetry. One sees that the target signals are well recovered by all the inversions. It is therefore unclear if nonsymmetry should be introduced during the inversion if the only available information is the flow rate. Considering the brachial pressures, still the improvement is not spectacular compared to the symmetric configuration as many combinations produce acceptable brachial pressure levels. A symmetric set up appears to be suitable for this patient. This is shown in Figure 24 indicating the acceptable nonsymmetry combinations for $\left(a_{R}, a_{C}\right)$, ie, the combinations for which similar results than the symmetric WK3 conditions are obtained. The same trends were observed for the other patients considered in this study. In short, if one wants the inversion to identify possible nonsymmetry, one would need more discriminating data. 

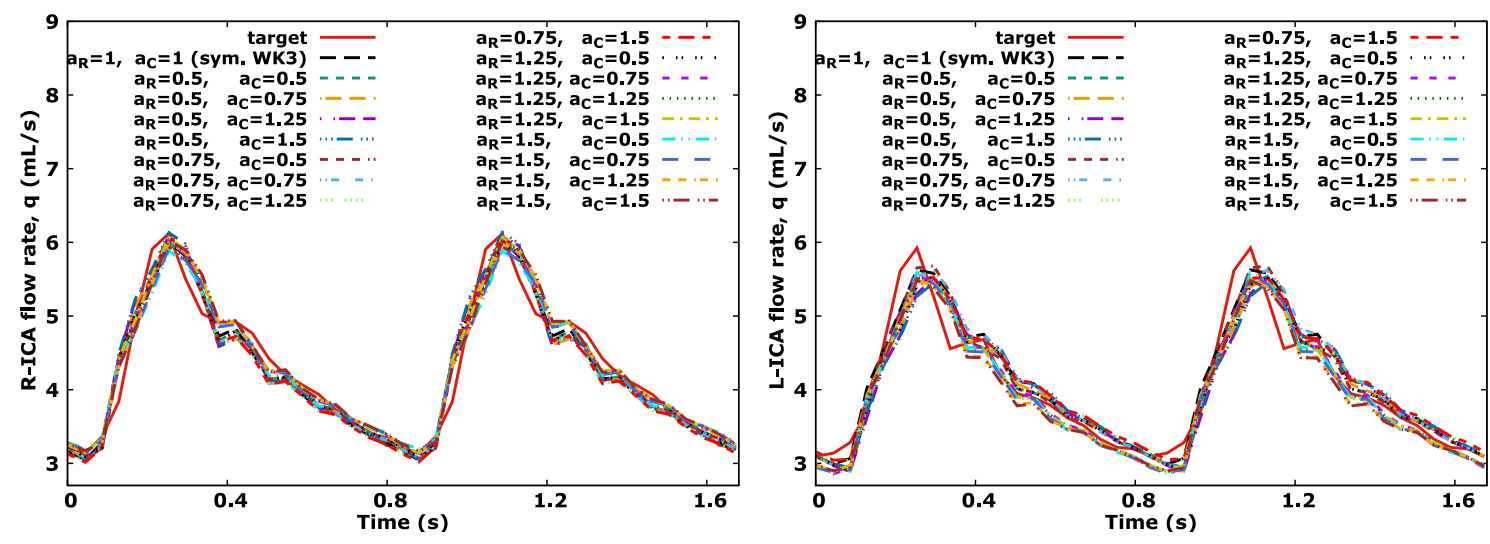

Figure 22. Comparison of the model simulated blood flow rate waveform after inversion with different combinations of $a_{C}$ and $a_{R}$.
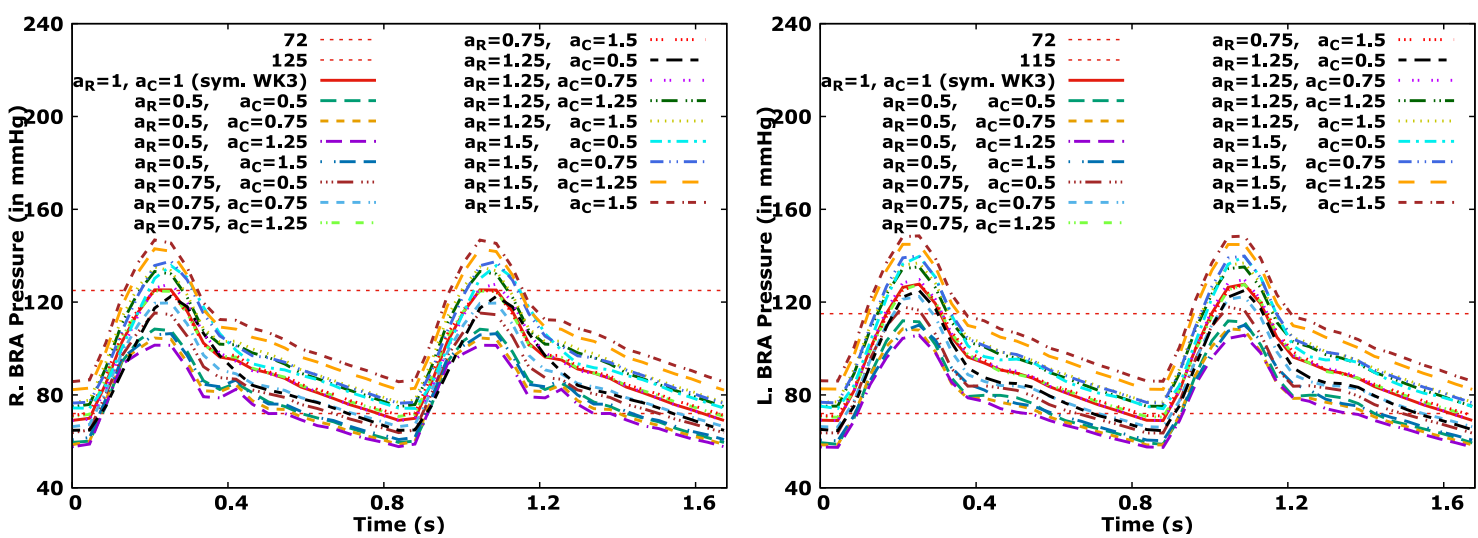

Figure 23. Comparison between R. and L. brachial pressures after inversion with different combinations of $a_{C}$ and $a_{R}$. 

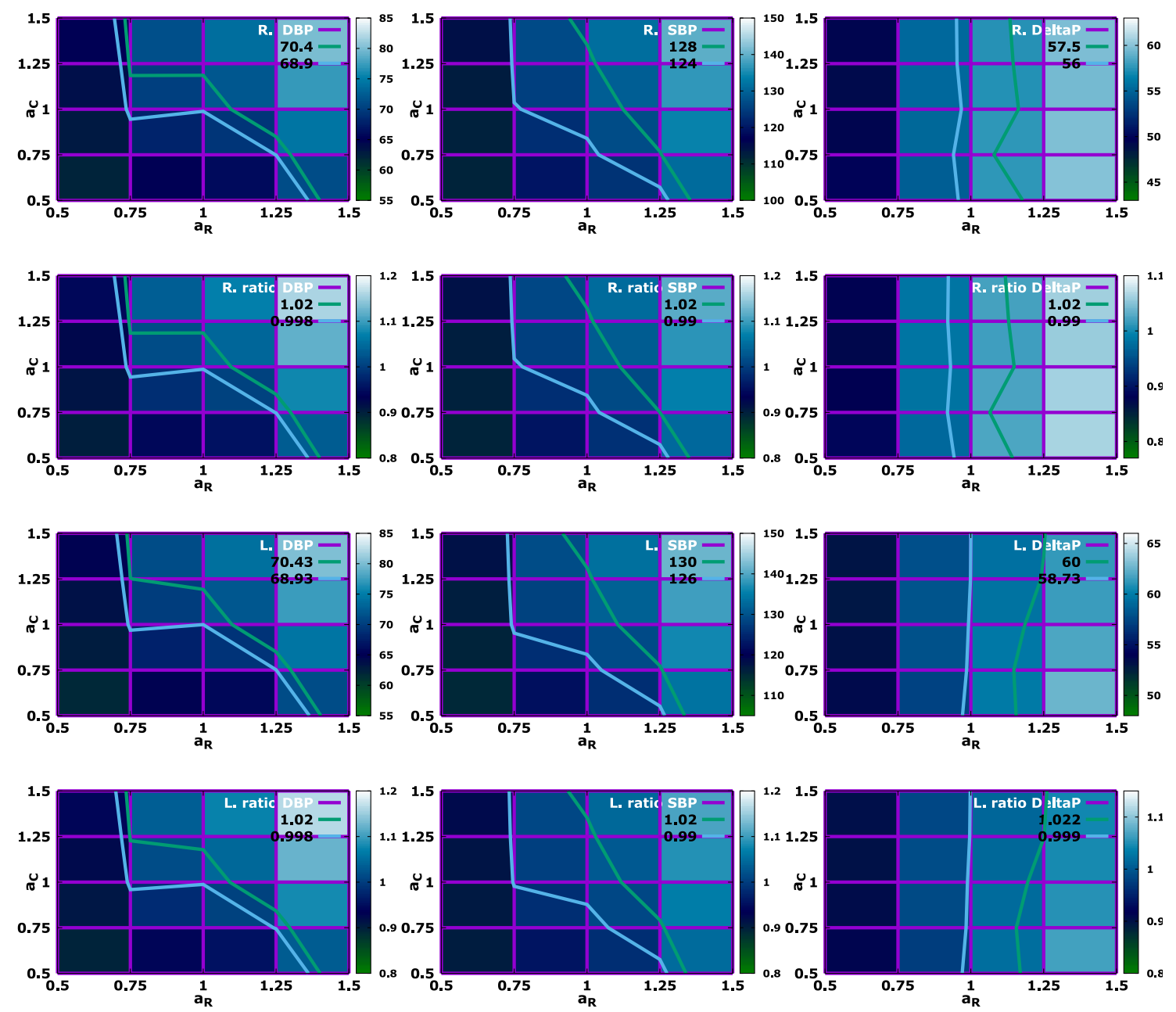

Figure 24. Impact of the nonsymmetry on compliances and resistances on the right $(\mathrm{R})$ and left $(\mathrm{L})$ brachial pressures. Diastolic (left column) and Systolic (middle column) pressures (DBP and SBP) are sensitive to nonsymmetry in both resistance and compliance while DeltaP, the difference between the two (right column) only sees the nonsymmetry in the resistance. The delimited region by the two contours indicates where the nonsymmetric combination produces similar results than the symmetric WK3 conditions.

\section{ALTERNATIVE BACKWARD UNCERTAINTY QUANTIFICATION}

\subsection{Linear theory for an alternative covariance matrix construction}

EnKF is an elegant way for backward uncertainty propagation as one has access to the covariance matrix $\left(\operatorname{cov}_{\mathbf{x}}\right)$ of the optimization variables during the inversion from ensemble information. Indeed, at each iteration $k$ of the EnKF algortihm, $\operatorname{cov}_{\mathbf{x}_{\mathbf{k}}}$ can be defined with a similar expression to those 
used for calculating the error covariance matrices necessary to the Kalman gain matrix computation:

$$
\operatorname{cov}_{\mathbf{x}_{\mathbf{k}}}=\frac{1}{q_{\text {ens }}-1} \sum_{i=1}^{q_{\text {ens }}}\left[\mathbf{x}_{k}^{\mathrm{f}_{i}}-\overline{\mathbf{x}}_{k}^{\mathrm{f}}\right]\left[\mathbf{x}_{k}^{\mathrm{f}_{i}}-\overline{\mathbf{x}}_{k}^{\mathrm{f}}\right]^{\top},
$$

where $q_{\text {ens }}$ is the size of the ensemble, the superscripts $\mathrm{f}$ and $\mathrm{f}_{i}$ stand for forecast and the $i$ th forecast member of an ensemble of size $q_{\mathrm{ens}}, \mathbf{x}_{k}^{\mathrm{f}_{i}}$ is the forecast ensemble of parameters for $i=1, \ldots, q_{\mathrm{ens}}$, and $\overline{\mathbf{x}}_{k}^{\mathrm{f}}$ is the mean of the forecast ensemble of parameters $\left(\overline{\mathbf{x}}_{k}^{\mathrm{f}}=\frac{1}{q_{\text {ens }}} \sum_{i=1}^{q_{\text {ens }}} \mathbf{x}_{k}^{\mathrm{f}_{i}}\right)$.

This section presents an alternative low-complexity construction of the covariance matrix [46] of the parameters $\left(\operatorname{cov}_{\mathbf{x}}\right)$ not necessitating ensemble information. It is based on the assumption of a local linear relationship ( $\delta \mathbf{y}=\mathcal{J} \delta \mathbf{x})$ between the hemodynamic parameter variations $\delta \mathbf{x}=\mathbf{x}-\overline{\mathbf{x}}$ and the state variations $\delta \mathbf{y}=\mathbf{y}-\overline{\mathbf{y}}$ with respect to the mean values $\overline{\mathbf{x}}$ and $\overline{\mathbf{y}}$. One interest of this construction is that it can also be used with deterministic minimization algorithms suitable for large dimensional problems where ensemble methods might fail.

Let us establish the expression for the covariance matrix $\operatorname{cov}_{\mathbf{x}}$ of the parameters $\mathbf{x}$ knowing the covariance matrix $\operatorname{cov}_{\mathbf{y}}$ of the model solution. These are supposed independent which means that $\operatorname{cov}_{\mathbf{y}}$ is diagonal.

The covariance matrix of $\mathbf{y}$ is given by

$$
\begin{aligned}
\operatorname{cov}_{\mathbf{y}}=\mathbb{E}\left[(\mathbf{y}-\overline{\mathbf{y}})(\mathbf{y}-\overline{\mathbf{y}})^{\top}\right]=\mathbb{E}\left[\mathcal{J}(\mathbf{x}-\overline{\mathbf{x}})(\mathbf{x}-\overline{\mathbf{x}})^{\top} \mathcal{J}^{\top}\right] & =\mathcal{J} \mathbb{E}\left[(\mathbf{x}-\overline{\mathbf{x}})(\mathbf{x}-\overline{\mathbf{x}})^{\top}\right] \mathcal{J}^{\top} \\
& =\mathcal{J} \operatorname{cov}_{\mathbf{x}} \mathcal{J}^{\top},
\end{aligned}
$$

and therefore,

$$
\operatorname{cov}_{\mathbf{x}}=\mathcal{J}^{-1} \operatorname{cov}_{\mathbf{y}} \mathcal{J}^{-\top}
$$

where $\mathcal{J}^{-\top}=\left(\mathcal{J}^{\top}\right)^{-1}$ and $\mathcal{J}=\nabla_{\mathbf{x}} \mathbf{y}$. As $\mathbf{x}$ and $\mathbf{y}$ do not have the same size, $\mathcal{J}$ is a rectangular matrix. A least square formulation is introduced to give a sense to $\mathcal{J}^{-1}$ through its normal form, minimizing

$$
\frac{1}{2}<\mathcal{J} \operatorname{cov}_{\mathbf{x}} \mathcal{J}^{\top}, \mathcal{J} \operatorname{cov}_{\mathbf{x}} \mathcal{J}^{\top}>-<\operatorname{cov}_{\mathbf{y}}, \mathcal{J} \operatorname{cov}_{\mathbf{x}} \mathcal{J}^{\top}>
$$

First order optimality condition with respect to $\operatorname{cov}_{\mathbf{x}}$ gives

$$
\mathcal{J}^{\top} \mathcal{J} \operatorname{cov}_{\mathbf{x}} \mathcal{J}^{\top} \mathcal{J}-\mathcal{J}^{\top} \operatorname{cov}_{\mathbf{y}} \mathcal{J}=0
$$


which implies

$$
\operatorname{cov}_{\mathbf{x}}=\left(\mathcal{J}^{\top} \mathcal{J}\right)^{-1} \mathcal{J}^{\top} \operatorname{cov}_{\mathbf{y}} \mathcal{J}\left(\mathcal{J}^{\top} \mathcal{J}\right)^{-1}
$$

and finally leads to

$$
\operatorname{cov}_{\mathbf{x}}=\mathcal{J}^{-1} \operatorname{cov}_{\mathbf{y}} \mathcal{J}^{-\top}=\left(\mathcal{J}^{\top} \operatorname{cov}_{\mathbf{y}}{ }^{-1} \mathcal{J}\right)^{-1}
$$

To get $\operatorname{cov}_{\mathbf{x}}$ knowing $\operatorname{cov}_{\mathbf{y}}$, it is therefore sufficient to evaluate the Jacobian $\mathcal{J}$. This can be done using a finite difference approximation.

When the inversion is successful, the model states $\mathbf{y}$ and the observations $\mathbf{y}_{\text {obs }}$ are close. Assuming that $\operatorname{cov}_{\mathbf{y}_{\mathrm{obs}}} \sim \operatorname{cov}_{\mathbf{y}}$, this construction from $\operatorname{cov}_{\mathbf{y}}$ can be extended to $\operatorname{cov}_{\mathbf{y}_{\mathrm{obs}}}$.

\subsection{Application to the patient-specific data}

This section applies the backward uncertainty quantification construction presented in Section 6.1 to the patient-specific data and compares the outcome with the ensemble analysis through EnKF results.

Figure 25 shows a comparative overview of the square root of $\mathbf{c o v}_{\mathbf{x}}$ diagonal elements calculated using EnKF (blue points) and the low cost linear theory (red points). These values represent standard deviation associated with the estimated parameters. The linear theory seems to provide lower bounds for the backward uncertainty on the inversion. This comparison shows that the linear hypothesis permits to a posteriori obtain the uncertainty on the inversion solution with an error of less than $15 \%$, and this especially without requiring any extra information when used together with a gradient based minimization algorithm. 


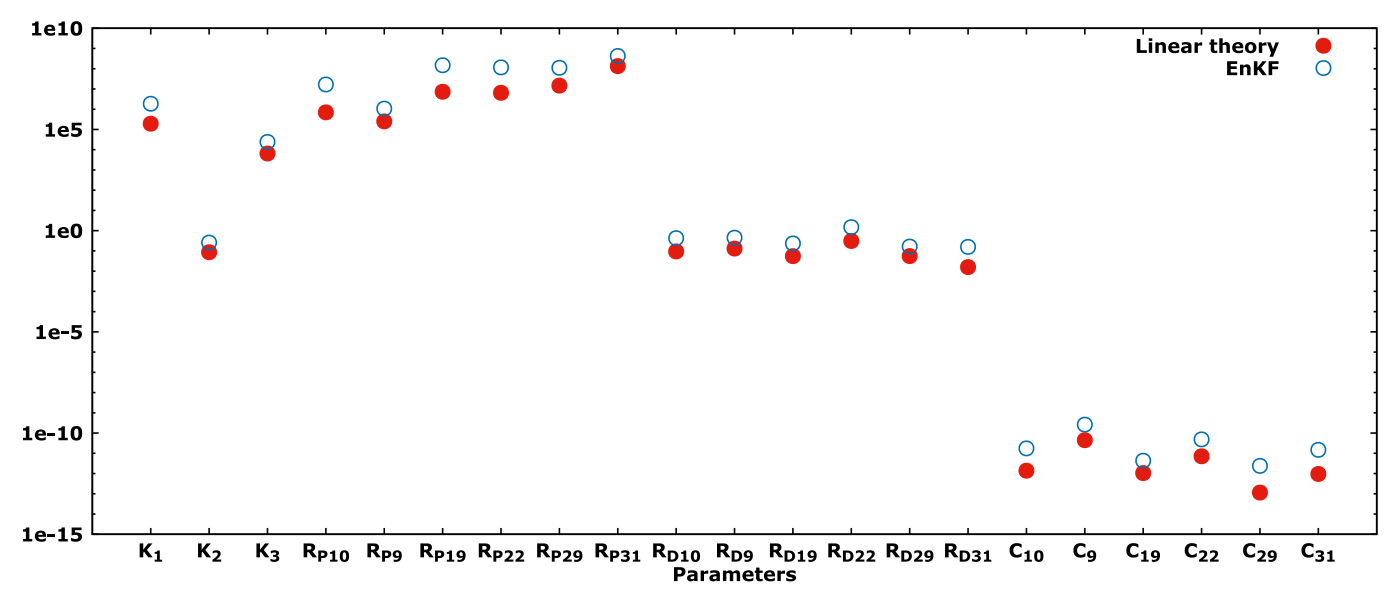

Figure 25. Comparison of the square root of $\operatorname{cov}_{\mathbf{x}}$ diagonal elements coming from EnKF solution (blue points) to the square root of $\operatorname{cov}_{\mathbf{x}}$ diagonal elements calculated with the low complexity deterministic evaluation based on the linear theory given in equation 8 (red points).

\section{LIMITATIONS}

It is worth bearing in mind the limitations of the present study, both intentional and unintentional. One of the limitations could be the use of OD models. Indeed, as pointed out by Shi et al [47] and Kokalari et al [48], the 0D models consider as uniform the distribution of fundamental variables (pressure, volume, and flow rate) at any instant in time. Thereby, they do not account for the spatial variation of these parameters [48]. They are therefore only appropriate for the study of global distribution of the pressure, flow rate, and blood volume in systemic arterial network and for specific physiological conditions. Phenomena, such as wave transmission, wave travel, and wave reflections, cannot be or are not studied [49]. It is worth noting that several studies have pointed out the shortcomings of the lumped models and have proposed more complex and sophisticated models to better capture the characteristics of blood flow [33, 47, 50-52]. However, this is not central to our discussion and is beyond the scope of this paper. What is shown here and is regularly employed in the literature concerning arterial hemodynamics is that this simple model permits to give reasonable results. Moreover, the region studied being compact, (the size of the cerebral network [of order 10 $\mathrm{cm}$ ] is small compared with typical pressure wavelength [of order a few meters]), assuming that all 
the quantities are homogeneous over each segment (viz, making the $0 \mathrm{D}$ assumption at the scale of each element), is acceptable. Of course, it would not be acceptable if this study was dealing with the arterial network of the whole body.

Another limitation could be the choice of the formula relating Young modulus, thickness, and radius for the cerebral arteries in Equation 5. This work assumes the same phenomenological relation for both large and cerebral arteries. The other factor might be the failure to account for the variation of arteries radii along arterial axes and the adaptation of missing data, such as the geometrical parameters of the arteries, from the literature. Future work should include sensitivity analysis of the optimization parameters outcomes with respect to this simplification. For instance, one way to quantify how this simplification would affect the results of optimization parameters is to perform several inversions, with the present tool, using perturbed geometries and see the impact of this perturbation on the outcomes. This is a step to be taken in the future to improve the robustness of the approach.

As possible improvements, one could also mention the marked changes in simulated brachial pressure wave resulting from assigning different resistances for left and right pairs of terminal cerebral compartments. From the physiological point of view, this might not be correct as some studies support that arterial blood pressure wave is mainly determined by the systemic vessels rather than the cerebral vessels. In other words, changing cerebral vascular resistances should not induce such large changes in systemic arterial pressure wave. To demonstrate the relevance of this issue, new tests of nonsymmetry in resistances with $a_{R}=1.5$ only have been performed. The results are summarized in Figure 26. They show that changing the resistances of only one termination in cerebral or brachial arteries has no effect on brachial pressure. However, they reveal that changing resistances at only thoracic aorta termination has the same impact (or even more) on brachial pressure as the nonsymmetry in resistances at all the arterial terminations except thoracic aorta case above presented. Furthermore, one notices that changing resistances at all terminations induces more important changes in brachial pressure. Thus, the apparent effects of resistances at arterial terminations on the brachial pressure might result from the fact that the lower part of 
systemic circulation, which is of major importance in determining arterial blood pressure wave is only represented here by distal boundary conditions.

At last, it is necessary to highlight the lack of clinically measured flow rates in arteries other than ICAs and ascending aorta. This prevents the proper validation of the predicted flow divisions among major tissues. More clinical data are required.
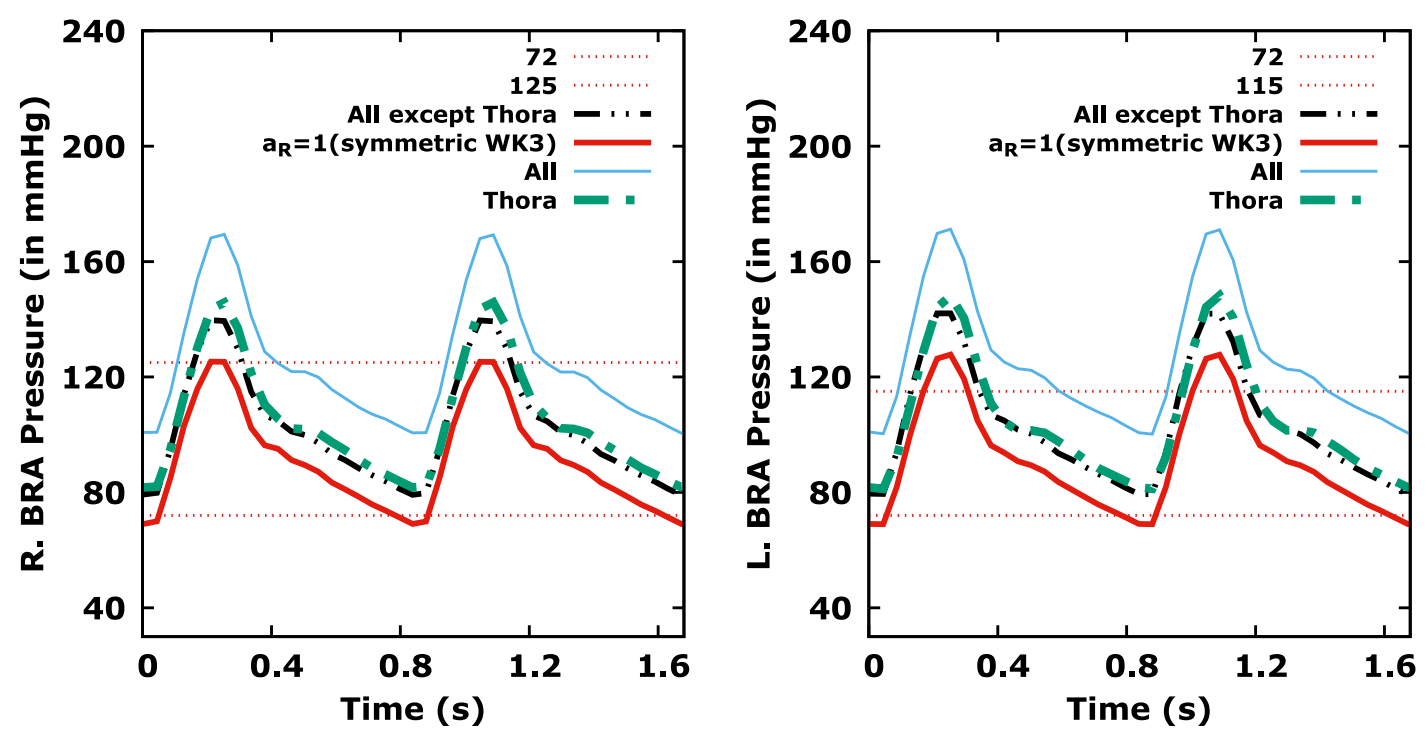

Figure 26. Importance of systemic arteries with respect to cerebral arteries in determining R. and L. brachial pressures estimated using WK3 with nonsymmetry in resistances with $a_{R}=1.5$ only. Changing resistances at only thoracic aorta termination (dashed green) has the same impact on brachial pressure as the nonsymmetry in resistances at all the arterial terminations except thoracic aorta case (dashed black). Changing resistances at all terminations (thin blue) induces more important changes in brachial pressure. The clinically measured SBP and DBP in the right and the left brachial artery are 125 and 72 , and 115 and 72 , respectively.

\section{CONCLUSION}

The robustness of a functional cerebral imaging platform has been analyzed for patient-specific situations. In particular, detailed results for one patient have been reported to illustrate the kind of information and sensitivity analyses which can be produced in addition to the classical inversion 
results. The aim is to be able to quantify the impact of the uncertainty of different parameters usually considered as deterministic on the hemodynamic parameters obtained after inversion.

An important source of uncertainty being the flow rate from the heart is that the sensitivity of the inversion with respect of the inlet flow has been analyzed. The conclusion drawn from this analysis has been that the predicted pressure and the elastic parameter estimation are of the same order of magnitude than the assumed uncertainty on the inlet flow rate. This provides additional guidance to practitioners as it gives some indication for the level of uncertainty one can tolerate because of manual acquisition steps. The sensitivity of the inversion with respect to the choice of the boundary conditions in the direct model has been analyzed. The WK3 boundary conditions appear necessary for a correct retrieval of the target signals. Then the impact of nonsymmetry in the terminations resistances and compliances has been analyzed. The former effects appear prominent. However, one notices that available observations are not enough discriminating and, therefore, do not make it obvious for possible nonsymmetry to be identified through the inversion procedure.

Finally, an ensemble approach might be unsuccessful if the size of the inversion problem is large. To address this issue, one might use deterministic gradient-based inversion algorithms together with an adjoint formulation for the gradient evaluation. But these algorithms do not propagate backward the uncertainty on the observations. To address this issue, a low-complexity backward uncertainty quantification construction has been introduced. It uses a local linear relationship between the blood flow rate and the hemodynamic parameters. The construction has been favorably compared to the ensemble outcome, which is interesting as this is a generic construction and can be used with any deterministic inversion algorithm.

\section{ACKNOWLEDGMENT}

The authors greatly thank Prof-Dr V. Costalat, Dr E. Le Bars and Dr J. Deverdun from the Department of Neuroradiology of the Centre Hospitalier Régional Universitaire de Montpellier, 
Gui de Chauliac, Montpellier, France, for providing the medical data and Dr J. Siguenza and Dr R.

Lal for helpful discussions. 


\section{REFERENCES}

1. World health organization 2018. cardiovascular diseases (cvds). The United Nations, accessed 24 february 2018 ; URL <http://Www.who.int/mediacentre/factsheets/ fs $317 / \mathrm{en} />$.

2. Hoksbergen A, Fülesdi B, Legemate D, Csiba L. Collateral configuration of the circle of willis transcranial color-coded duplex ultrasonography and comparison with postmortem anatomy. Stroke 2000; 31(6):1346-1351.

3. Brain aneurysm foundation 2018. about brain aneurysms. Brain Aneurysm Foundation, accessed 09 april 2018 ; URL https://www.bafound. org/about-brain-aneurysms/brain-aneurysm-basics/ warning-signs-symptoms/.

4. Taylor CL, Yuan Z, Selman WR, Ratcheson RA, Rimm AA. Cerebral arterial aneurysm formation and rupture in 20,767 elderly patients: hypertension and other risk factors. Journal of neurosurgery 1995; 83(5):812-819.

5. Costalat V, Sanchez M, Ambard D, Thines L, Lonjon N, Nicoud F, Brunel H, Lejeune J, Dufour $\mathrm{H}$, Bouillot $\mathrm{P}$, et al. Biomechanical wall properties of human intracranial aneurysms resected following surgical clipping (irras project). Journal of biomechanics 2011; 44(15):2685-2691.

6. Sanchez M, Ambard D, Costalat V, Mendez S, Jourdan F, Nicoud F. Biomechanical assessment of the individual risk of rupture of cerebral aneurysms: a proof of concept. Annals of biomedical engineering 2013; 41(1):28-40.

7. Sanchez M, Ecker O, Ambard D, Jourdan F, Nicoud F, Mendez S, Lejeune JP, Thines L, Dufour $\mathrm{H}$, Brunel $\mathrm{H}$, et al. Intracranial aneurysmal pulsatility as a new individual criterion for rupture risk evaluation: Biomechanical and numeric approach (irras project). American Journal of Neuroradiology 2014; 35(9):1765-1771, doi:10.3174/ajnr.A3949. URL http: //www.ajnr.org/content/35/9/1765. 
8. Etminan N, Buchholz BA, Dreier R, Bruckner P, Torner JC, Steiger HJ, HÃd'nggi D, Loch MR. Cerebral aneurysms: Formation, progression and developmental chronology. Translational stroke research 2014; 2(5):167-173, doi:10.1007/s12975-013-0294-х.

9. Mohan D, Munteanu V, Coman T, Ciurea A. Genetic factors involves in intracranial aneurysmsactualities. Journal of medicine and life 2015; 8(3):336.

10. Alastruey J, Parker K, Peiró J, Byrd S, Sherwin S. Modelling the circle of willis to assess the effects of anatomical variations and occlusions on cerebral flows. Journal of biomechanics 2007; 40(8):1794-1805.

11. Kayembe KN, Sasahara M, Hazama F. Cerebral aneurysms and variations in the circle of willis. American Heart Association, Inc. 1984; 15(5):846-850, doi:10.1161/01.STR.15.5.846. URL http://stroke.ahajournals.org/content/15/5/846.

12. Reymond P, Merenda F, Perren F, Rüfenacht D, Stergiopulos N. Validation of a one-dimensional model of the systemic arterial tree. American Journal of Physiology-Heart and Circulatory Physiology 2009; 297(1):H208-H222.

13. Mulder G, Bogaerds A, Rongen P, van de Vosse F. The influence of contrast agent injection on physiological flow in the circle of willis. Medical engineering \& physics 2011; 33(2):195-203.

14. Karmonik C, Yen C, Grossman RG, Klucznik R, Benndorf G. Intra-aneurysmal flow patterns and wall shear stresses calculated with computational flow dynamics in an anterior communicating artery aneurysm depend on knowledge of patient-specific inflow rates. Acta Neurochirurgica May 2009; 151(5):479-485, doi:10.1007/s00701-009-0247-z. URL https : //doi.org/10.1007/s00701-009-0247-z.

15. Hassan T, Hassan AA, Ahmed YM. Influence of parent vessel dominancy on fluid dynamics of anterior communicating artery aneurysms. Acta Neurochirurgica Feb 2011; 153(2):305-310, doi:10.1007/s00701-010-0824-1. URL https://doi .org/10.1007/ s00701-010-0824-1. 
16. Lazzaro MA, Ouyang B, Chen M. The role of circle of willis anomalies in cerebral aneurysm rupture. Journal of NeuroInterventional Surgery 2012; 4(1):22-26, doi:10.1136/jnis.2010. 004358. URL https: / / jnis.bmj.com/content/4/1/22.

17. G HP, O ML. Simulation of one-dimensional blood flow in networks of human vessels using a novel tvd scheme. International Journal for Numerical Methods in Biomedical Engineering 2015; 31(5):e02 701, doi:10.1002/cnm.2701. URL https://onlinelibrary.wiley • com/doi/abs/10.1002/cnm.2701,e02701 CNM-Sep-14-0153.R1.

18. Flores BC, Scott WW, Eddleman CS, Batjer HH, Rickert KL. The a1-a2 diameter ratio may influence formation and rupture potential of anterior communicating artery aneurysms. Neurosurgery 2013; 73(5):845-853, doi:10.1227/NEU.0000000000000125. URL http:// dx.doi.org/10.1227/NEU.0000000000000125.

19. Liang F, Liu X, Yamaguchi R, Liu H. Sensitivity of flow patterns in aneurysms on the anterior communicating artery to anatomic variations of the cerebral arterial network. Journal of Biomechanics Nov 2016; 49(15):3731-3740, doi:doi:10.1016/j.jbiomech.2016.09.031. URL https://doi.org/10.1016/j.jbiomech.2016.09.031.

20. Bertoglio C, Moireau P, Gerbeau JF. Sequential parameter estimation for fluid-structure problems: Application to hemodynamics. International Journal for Numerical Methods in Biomedical Engineering 2012; 28(4):434-455.

21. Moireau P, Bertoglio C, Xiao N, Figueroa CA, Taylor C, Chapelle D, Gerbeau JF. Sequential identification of boundary support parameters in a fluid-structure vascular model using patient image data. Biomechanics and modeling in mechanobiology 2013; 12(3):475-496.

22. Lombardi D. Inverse problems in 1d hemodynamics on systemic networks: A sequential approach. International journal for numerical methods in biomedical engineering 2014; 30(2):160-179. 
23. Chabiniok R, Moireau P, Lesault PF, Rahmouni A, Deux JF, Chapelle D. Estimation of tissue contractility from cardiac cine-mri using a biomechanical heart model. Biomechanics and modeling in mechanobiology 2012; 11(5):609-630.

24. Caiazzo A, Caforio F, Montecinos G, Muller LO, Blanco PJ, Toro EF. Assessment of reduced-order unscented kalman filter for parameter identification in 1-dimensional blood flow models using experimental data. International Journal for Numerical Methods in Biomedical Engineering aug 2017; 33(8):e2843, doi:10.1002/cnm.2843. URL https:// onlinelibrary.wiley.com/doi/abs/10.1002/cnm.2843, e2843 cnm.2843.

25. Lal R, Mohammadi B, Nicoud F. Data assimilation for identification of cardiovascular network characteristics. International Journal for Numerical Methods in Biomedical Engineering may 2017; 33(5):e2824, doi:10.1002/cnm.2824. URL https://onlinelibrary.wiley. com/doi/abs/10.1002/cnm.2824, e2824 cnm.2824.

26. Lal R. Data assimilation and uncertainty quantiiňĄcation in cardiovascular biomechanics. $\mathrm{PhD}$ Thesis, University of Montpellier, Montpellier, France 2017.

27. Mohammadi B. Backward uncertainty propagation in shape optimization. Int. J. for Numerical Methods in Fluids 2016; 103(4)-DOI: 10.1002/fld.4077:307-323.

28. Mohammadi B, OPironneau. Applied Shape Optimization for Fluids (2nd Edition). Oxford Univ. Press: Oxford, 2009.

29. Quarteroni A, Tuveri M, Veneziani A. Computational vascular fluid dynamics: problems, models and methods. Computing and Visualization in Science 2000; 2(4):163-97, doi:10.1007/ s007910050039.

30. Kiselev I, Semisalov B, Sharipov R, Kolpakov F. Modular modeling of the human cardiovascular system, vol. 7. Nova Science Publishers Inc., 2012.

31. Liang F, Takagi S, Himeno R, Liu H. Multi-scale modeling of the human cardiovascular system with applications to aortic valvular and arterial stenoses. Medical \& Biological Engineering \& 
Computing Jul 2009; 47(7):743-755, doi:10.1007/s11517-009-0449-9. URL https : / / doi . org/10.1007/s11517-009-0449-9.

32. Ursino M, Giannessi M. A model of cerebrovascular reactivity including the circle of willis and cortical anastomoses. Annals of biomedical engineering 2010; 38(3):955-974.

33. Quarteroni A, Ragni S, Veneziani A. Coupling between lumped and distributed models for blood flow problems. Computing and Visualization in Science 2001; 4(2):111-124.

34. Milišić V, Quarteroni A. Analysis of lumped parameter models for blood flow simulations and their relation with 1d models. ESAIM: Mathematical Modelling and Numerical Analysis 2004; 38(4):613-632.

35. Pant S, Fabrèges B, Gerbeau JF, Vignon-Clementel I. A methodological paradigm for patientspecific multi-scale cfd simulations: from clinical measurements to parameter estimates for individual analysis. International journal for numerical methods in biomedical engineering 2014; 30(12):1614-1648.

36. Olufsen MS, Nadim A, et al.. On deriving lumped models for blood flow and pressure in the systemic arteries. Math Biosci Eng 2004; 1(1):61-80.

37. Boileau E, Nithiarasu P, Blanco PJ, MÃijller LO, Fossan FE, Hellevik LR, Donders WP, Huberts W, Willemet M, Alastruey J. A benchmark study of numerical schemes for onedimensional arterial blood flow modelling. International Journal for Numerical Methods in Biomedical Engineering oct 2015; 31(10):e02 732, doi:10.1002/cnm.2732. URL https: / / onlinelibrary.wiley.com/doi/abs/10.1002/cnm.2732, e02732 cnm.2732.

38. Byrne GD, Hindmarsh AC. Stiff ode solvers: A review of current and coming attractions. Journal of Computational physics 1987; 70(1):1-62.

39. SP Corwin ST, White S. Solving odes and ddes with impulses. JNAIAMJ.Numer.Anal.Indust.Appl.Math 2008; 3:139-149. 
40. Olufsen MS. Structured tree outflow condition for blood flow in larger systemic arteries. American journal of physiology-Heart and circulatory physiology 1999; 276(1):H257-H268.

41. Stergiopulos N, Young D, Rogge T. Computer simulation of arterial flow with applications to arterial and aortic stenoses. Journal of biomechanics 1992; 25(12):1477-1488.

42. Gul R. Mathematical modeling and sensitivity analysis of lumped-parameter model of the human cardiovascular system. PhD Thesis in Freien Universität Berlin 01 2016; URL https : //www.researchgate.net/publication/301852867_Mathematical_ Modeling_and_Sensitivity_Analysis_of_Lumped-Parameter_Model_ of_the_Human_Cardiovascular_System.

43. Gul R, SchÃijtte C, Bernhard S. Mathematical modeling and sensitivity analysis of arterial anastomosis in the arm. Applied Mathematical Modelling 2016; 40(17):7724 - 7738, doi: https://doi.org/10.1016/j.apm.2016.03.041. URL http://www.sciencedirect.com/ science/article/pii/s0307904X16301895.

44. Liang F, Fukasaku K, Liu H, Takagi S. A computational model study of the influence of the anatomy of the circle of willis on cerebral hyperperfusion following carotid artery surgery. Biomedical engineering online 09 2011; 10:84.

45. Schollenberger J. A lumped parameter model of cerebral blood flow regulation: Applications to simulation of carotid endarterectomy. Technische Universitat Munchen, Master Thesis 06 2015; URL https://bloodflow.engin.umich.edu/wp-content/uploads/sites/ 165/2014/07/20141201_thesis.pdf.

46. Wasserman L. All of Statistics: A Concise Course in Statistical Inference. ISBN 0-387-40272-1, Springer, 2004.

47. Shi Y, Lawford P, Hose R. Review of zero-d and 1-d models of blood flow in the cardiovascular system. Biomedical engineering online 04 2011; 10:33. 
48. Kokalari I, Karaja T, Guerrisi M. Review on lumped parameter method for modeling the blood flow in systemic arteries. Journal of Biomedical Science and Engineering 2013; 6(1):92-99, doi:10.4236/jbise.2013.61012. URL http://file.scirp.org/Html/12-9101565_ $27458 \cdot$ htm.

49. Westerhof N, Lankhaar JW, Westerhof BE. The arterial windkessel. Medical \& Biological Engineering \& Computing Feb 2009; 47(2):131-141, doi:10.1007/s11517-008-0359-2. URL https://doi.org/10.1007/s11517-008-0359-2.

50. Formaggia L, Gerbeau JF, Nobile F, Quarteroni A. On the coupling of $3 \mathrm{~d}$ and $1 \mathrm{~d}$ navier-stokes equations for flow problems in compliant vessels. Computer Methods in Applied Mechanics and Engineering 12 2001; 191:561-582.

51. Vignon-Clementel IE, Figueroa CA, Jansen KE, Taylor CA. Outflow boundary conditions for three-dimensional finite element modeling of blood flow and pressure in arteries. Computer Methods in Applied Mechanics and Engineering 2006; 195(29):3776 - 3796, doi:https://doi. org/10.1016/j.cma.2005.04.014. URL http://www.sciencedirect.com/science/ article/pii/S0045782505002586, absorbing Boundary Conditions.

52. Kim HJ, Vignon-Clementel IE, Figueroa CA, LaDisa JF, Jansen KE, Feinstein JA, Taylor CA. On coupling a lumped parameter heart model and a three-dimensional finite element aorta model. Annals of Biomedical Engineering Nov 2009; 37(11):2153-2169, doi:10.1007/ s10439-009-9760-8. URL https: //doi.org/10.1007/s10439-009-9760-8. 